\title{
Resource Allocation in Elastic Optical Networks with Physical-Layer Impairments
}

\author{
A Thesis \\ Presented to \\ the faculty of the School of Engineering and Applied Science \\ University of Virginia

\begin{abstract}
in partial fulfillment
of the requirements for the degree

Master of Science
\end{abstract} \\ by \\ Yuxin Xu \\ August 2017
}




\section{APPROVAL SHEET}

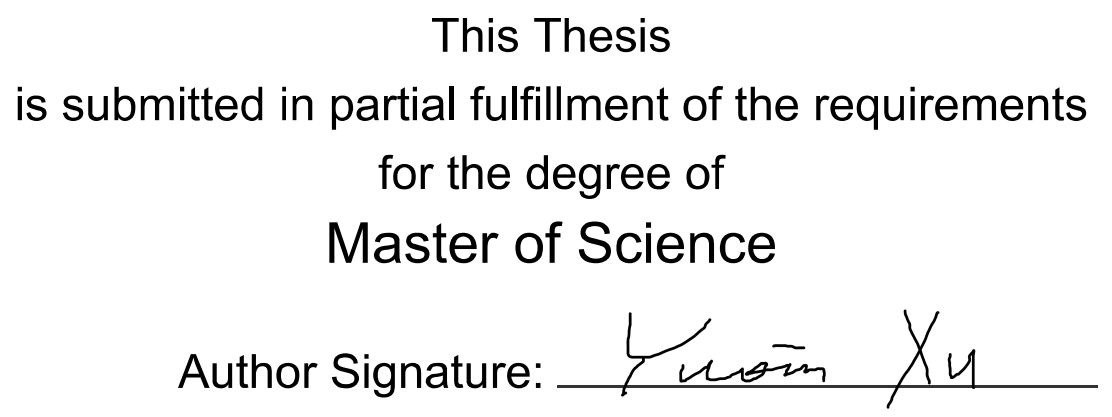

This Thesis has been read and approved by the examining committee:

Advisor: Maite Brandt-Pearce

Committee Member: Stephen G. Wilson

Committee Member: Farzad Farnoud

Committee Member:

Committee Member:

Committee Member:

Accepted for the School of Engineering and Applied Science:

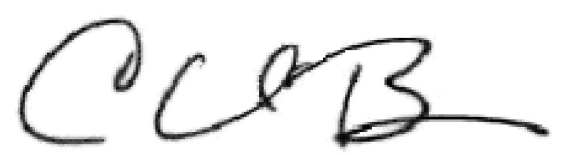

Craig H. Benson, School of Engineering and Applied Science

August 2017 


\section{Acknowledgements}

First, I would like to gratefully thank my advisor, Professor Maite Brandt-Pearce for her guidance and patience. Her continuous encouragement and profound knowledge greatly helped me during my graduate studies at the University of Virginia. Her dedicated and rigorous attitude is the guidance of my future career.

Second, I would like to thank my families, Ruilin Zhu, Zhen Jiang, Ning Xu and Weili Zhu, for their love, trust and understanding. They have done nothing but love me whole-heartedly.

Third, I would like to thank my friends, Xi Chen, Yifei Wang, Jiashen Zhang, Jiahui Zhu, Aaron Cho, Alex Park and Yalan Wu for their unconditional support and endless love.

Lastly, I am also grateful to Li Yan, Jie Lian, Jesse Morgan, Stephen Wilson, Toby Berger and many people who helped with my studies and research. 


\section{Abstract}

Elastic optical networks (EONs) have been proposed to meet future communication demands [1]. Planning the resource usage of EONs has been the subject of extensive research. Routing and spectrum assignment (RSA) algorithms are used to minimize the network resources used. Estimation of physical-layer impairments (PLIs) in EONs plays an important role in the network planning stage. The transmission reach (TR) model and the Gaussian noise (GN) model are broadly considered in the estimation of the PLIs. However, due to the nature of these models, their performance remains problematic. Thus, based on the GN model, this thesis proposes a physical layer estimation model, referred to as the conservative linearized Gaussian noise (CLGN) model. In addition, we improve upon the existing TR model with a novel algorithm for obtaining the parameters, leading to a fairer comparison between the TR model and the CLGN model. We then introduce a link-based mixed integer linear programming (MILP) formulation to address the RSA problem to quantify the performance of each PLI model. Suffering from the large computational burden brought by the MILP, we propose a heuristic algorithm, referred to as the sequential allocation (SA) algorithm. The SA algorithm can solve a large number of demands in a large scale network with a reasonable computational burden. Lastly, we show through simulation that network resources such as spectrum and regeneration nodes can be saved by utilizing the CLGN model, compared with the TR model. We also show that the SA algorithm has notably better optimization solutions, compared with a published algorithm, the recursive MILP [2]. Moreover, we also show that our proposed system, which is based on the CLGN model and the SA algorithm, speeds up the optimization process and provides similar resource usage, compared to the published benchmark system in [3]. 


\section{Contents}

1 Introduction 1

1.1 Background and Motivation ................. 1

1.2 Literature Review . . . . . . . . . . . . . . . . . . . . . . . 3

1.3 Thesis Outline . . . . . . . . . . . . . . . . 5

2 EON Description and Problem Formulation $\quad 6$

2.1 Elastic Optical Network . . . . . . . . . . . . . . . 6

2.2 Gaussian Noise Model and Quality of Transmission . . . . . . . . . 8

2.3 Transmission Reach Model . . . . . . . . . . . . . . . . . . 11

2.4 Signal Regeneration . . . . . . . . . . . . . . . . . . 12

2.5 Routing and Spectrum Allocation (RSA) Problem . . . . . . . . . . 13

2.6 Heuristic Method . . . . . . . . . . . . . . . . . . . 14

3 Conservative Linearized GN Model $\quad 16$

3.1 Conservative Linearized Gaussian Noise (CLGN) Model . . . . . . . . 16

3.2 Gaussian Noise Based Transmission Reach (GNTR) Model . . . . . . 18

3.3 Link Level Analysis . . . . . . . . . . . . . . . . . . . . . . . . 22

4 Heuristic Algorithm for RSA in EONs 29

4.1 Notation for the Basic MILP . . . . . . . . . . . . . . . . 29 
4.2 Basic MILP Constraints . . . . . . . . . . . . . . . . . . . 31

4.3 QoT Requirements . . . . . . . . . . . . . . . . . . 32

4.3.1 GNTR Model . . . . . . . . . . . . . . . . . . . 32

4.3 .2 CLGN Model . . . . . . . . . . . . . . . . . . 33

4.4 MILP with Regeneration Nodes . . . . . . . . . . . . . . . . . 34

4.4.1 GNTR Model with Regeneration Nodes . . . . . . . . . . . . . 35

4.4.2 CLGN Model with Regeneration Nodes . . . . . . . . . . . . . 38

4.5 Heuristic Algorithm: Sequential Allocation . . . . . . . . . . . . . 39

4.5.1 Motivation of the Sequential Allocation . . . . . . . . . . . 39

4.5.2 SA Process ....................... . . . 41

4.6 Comparison with the Benchmark . . . . . . . . . . . . . 44

5 Numerical Results $\quad 47$

5.1 Simulation Settings . . . . . . . . . . . . . . . 47

5.2 Results for DT-14 Network . . . . . . . . . . . . . . . . . . . 48

5.3 Results for NSF-24 Network . . . . . . . . . . . . . . . . 51

5.3.1 RSA with Multi-Optimization Objectives . . . . . . . . 51

5.3.2 Comparison with the Recursive MILP . . . . . . . . . . . . 60

6 Conclusions and Future Work $\quad 64$

6.1 Conclusions ......................... 64

6.2 Future Work . . . . . . . . . . . . . . . . 65 


\section{List of Figures}

3.1 Illustration of interfering demands positioned in the optical spectrum.

3.2 NLI PSD per span versus the number of demands shared on the same fiber link, $M_{c}$, filling the $4000 \mathrm{GHz}$ spectrum. . . . . . . . . . . . 21

3.3 Illustration of the worst case interference on test demand $i$. . . . . . 21

3.4 Comparison of the transmission reach generated by the GNTR, the CLGN, and the GN models, for various $M_{c}$, with BPSK modulation.

3.5 Comparison of the transmission reach generated by the GNTR, the CLGN, and the GN models, for various $M_{c}$, with QPSK modulation.

3.6 Comparison of the estimation error $\left(\operatorname{Err}_{*}\right)$ generated by the GNTR, the CLGN, and the GN models, for various $M_{c}$. . . . . . . . 25

3.7 Histogram of normalized noise level in BPSK with various $M_{c}$. . . . 26

3.8 Histogram of transmission reach in BPSK with various $M_{c} \ldots \ldots$

3.9 Histogram of normalized noise level in QPSK with various $M_{c}$. . . . 27

3.10 Histogram of transmission reach in QPSK with various $M_{c} \ldots \ldots$. . 27

4.1 Six node test network [4]. The number on each link corresponds to the number of spans. . . . . . . . . . . . . . . 
4.2 Total spectrum usage versus number of demands for optimal MILP and proposed heuristic algorithm SA in 6-node test network, with BPSK modulation. (a) GNTR model. (b) CLGN model. . . . . . . . . . .

5.1 DT-14 network [4]. The number on each link corresponds to the number of spans. . . . . . . . . . . . . . . . . . . . . 48

5.2 NSF-24 network [2]. The number on each link represents the physical length of the link in $\mathrm{km} . \ldots \ldots \ldots \ldots$

5.3 (a) Total spectrum usage versus number of demands for proposed system and benchmark system in DT-14 network, with BPSK modulation. (b) Elapsed time versus number of demands for proposed system and benchmark system in DT-14 network. . . . . . . . . . . . . . .

5.4 (a) Optimization objective $C+\varepsilon T$ versus number of demands in NSF-24 network, with QPSK modulation. (b) Optimization objective $T+\varepsilon C$ versus number of demands in NSF-24 network, with QPSK modulation. 53

5.5 Total spectrum usage versus number of demands with different optimization objectives $C+\varepsilon T$ and $T+\varepsilon C$ in NSF-24 network. (a) BPSK modulation. (b) QPSK modulation. . . . . . . . . . . . .

5.6 (a) Number of regeneration nodes versus number of demands with different optimization objectives $C+\varepsilon T$ and $T+\varepsilon C$ in NSF-24 network, with QPSK modulation. (b) Number of regeneration circuits versus number of demands with different optimization objectives $C+\varepsilon T$ and $T+\varepsilon C$ in NSF-24 network, with QPSK modulation. . . . . . . . 
5.7 (a) Total spectrum usage versus number of demands with limited regeneration nodes, i.e., $T \leq 2$ and without limitation of regeneration nodes in NSF-24, with QPSK modulation. (b) Number of regeneration circuits versus number of demands with limited regeneration nodes, i.e., $T \leq 2$ and without limitation of regeneration nodes in NSF-24, with QPSK modulation. . . . . . . . . . . . . . . . 59

5.8 Total spectrum usage versus the number of demands for the SA and the re-MILP in NSF-24, with QPSK modulation. . . . . . . . . . 61

5.9 The number of regeneration nodes versus the number of demands for the SA and the re-MILP in NSF-24, with QPSK modulation. . . . .

5.10 The number of regeneration circuits versus the number of demands for the SA and the re-MILP in NSF-24, with QPSK modulation. . . . . 


\section{List of Tables}

2.1 Modulation Format, Spectral Efficiency and Threshold SINR (pre-FEC BER = $\left.4 \times 10^{-3}\right)[5] \ldots \ldots \ldots \ldots \ldots \ldots$

3.1 Fiber Parameters $[6]$. . . . . . . . . . . . . . . . . . 20 


\section{Acronyms}

ASE: Amplified spontaneous emission

BER: $\quad$ Bit error rate

BPSK: $\quad$ Binary phase shift keying

BVT: $\quad$ Bandwidth variable transceivers

CLGN: Conservative linearized Gaussian noise

DWDM: Dense Wavelength division multiplexing

EDFA: $\quad$ Erbium-doped fiber amplifier

EON: $\quad$ Elastic optical network

FEC: $\quad$ Forward error correction

GN: Gaussian noise

GNTR: Gaussian-noise-based transmission reach

ILP: Integer linear programming

MILP: $\quad$ Mixed integer linear programming

NLI: $\quad$ Nonlinear interference

OEO: Optical-electrical-optical

OFDM: Orthogonal frequency-division multiplexing

PLI: $\quad$ Physical layer impairments

PM: $\quad$ Polarization multiplexing

PSD: $\quad$ Power spectral density

QoT: Quality of transmission

QPSK: Quadrature phase-shift keying

Re-MILP: Recursive mixed integer linear programming 
ROADM: Reconfigurable optical add-drop multiplexers

RSA: Routing and spectrum assignment

RWA: Routing and wavelength assignment

SA: Sequential allocation

SCI: $\quad$ Self-channel interference

SINR: Signal to interference plus noise ratio

TR: $\quad$ Transmission reach

XCI: Cross-channel interference 


\section{Chapter 1}

\section{Introduction}

\subsection{Background and Motivation}

With the enormous growth of the communication industry and traffic heterogeneity, the next generation of long-haul elastic optical networks (EONs) has been proposed (motivated from dense wavelength division multiplexing (DWDM) networks) to meet future communication demands [1].

In accordance with the industry standard ITU G.694 [7], 88 channels, each channel spaced $50 \mathrm{GHz}$ apart, are supported by dense wavelength division multiplexing (DWDM) networks. In the DWDM network, multiple demands are accommodated in $50 \mathrm{GHz}$ frequency slots with slightly different center frequencies. Because the conventional DWDM network uses a fixed grid of $50 \mathrm{GHz}$ between two adjacent frequency intervals [8], the optical spectrum supporting data rates beyond $100 \mathrm{~Gb} / \mathrm{s}$ using standard modulation does not fit in the $50 \mathrm{GHz}$ ITU grid [1]. Therefore, DWDM networks are not able to satisfy the growing demands of communications. Consequently, EONs are proposed to meet the requirements of the next generation of communications. Unlike conventional DWDM networks, EONs can use bandwidth variable transceivers 
(BVT), making them suitable for heterogeneous traffic demands. Intrinsically, EONs use the continuous flexible optical bandwidth by partitioning the bandwidth into infinitely many frequency slots with the infinitely small granularity, resulting in the network bandwidth appearing elastic and continuous [4]. Without the limitation of the $50 \mathrm{GHz}$ ITU grid, EONs would be able to switch the broader spectrum channels in order to support high bit rate (such as $400 \mathrm{~Gb} / \mathrm{s}$ or $1 \mathrm{~Tb} / \mathrm{s}$ ) demands $[1,9]$. Hence, EONs are considered to be broadly applicable in the future.

However, the resources needed to build EONs (spectrum, regeneration nodes, optical amplifiers, etc.) are limited. Planning the resource usage of EON, the so-called routing and spectrum allocation (RSA) problem, has been the subject of extensive research $[3-5,10-12]$. This thesis proposes a series of algorithms that are able to reduce the network resources needed to implement continental-scale EONs.

Physical-layer impairments (PLIs) such as fiber loss, dispersion and nonlinearities can impair the quality of transmission (QoT) in long-haul networks [5]. The QoT identifies the network's capability of recovering the transmitted information. PLIs of EONs have been studied for the past several years. Estimation of the PLIs plays an important role in the network stage planning [13-15]. The most common model for estimating the PLIs is the transmission reach (TR) model [13], which approximates the maximum distance a signal can travel without regeneration. However, the TR model lacks sufficient flexibility and accuracy. This model estimates the worst case PLIs instead of considering the real-time network state. When we apply the TR model in realistic scenarios of routing and spectrum allocation for EONs, it severely overestimates the PLIs. In order to obtain a more accurate estimate of channel PLIs, a state-dependent model, the Gaussian noise (GN) model [11,15], has been proposed. However, the GN model is nonconvex and suffers from nonlinearities and complexity, making it less usable when applied to the RSA problem for EONs. Thus, we propose 
a linearized GN model to overcome the nonlinearity and complexity of the standard GN model.

The complexity of the RSA problem itself increases exponentially as the network dimensions expand. The optimal method of solving the RSA problem is to use a mixed integer linear programming (MILP). MILP engines do not perform well on large dimension networks and consequently cannot find the optimal solution within a reasonable time [16]. Therefore, in order to overcome this shortcoming, heuristic algorithms have been proposed to provide a sub-optimal solution within a reasonable time. Scalability, near-optimality, and time-consumption remain a problem for heuristic algorithms in published literature $[2-4,17]$. Therefore, we propose a heuristic algorithm, the sequential allocation (SA) algorithm, that performs at relatively high speeds, works with different PLI models, and has superb performance. The SA algorithm is capable of solving the RSA problem for large network topologies and traffic dimensions.

In summary, our proposed work applies a linearization of the GN model to estimate the PLIs of EONs, and solves the RSA problem through the application of the SA algorithm. Our work not only provides a significant saving of resources, but also solves the RSA problem in a reasonably short time. The high scalability as well as the close-to-optimal output of the proposed technique makes it suitable for practical networks.

\subsection{Literature Review}

In 1995, R. Ramaswami et al. published research on the routing and wavelength assignment (RWA) problem in fixed grid transparent networks [18]. The RWA problem is modeled as an integer linear programming (ILP) in a WDM optical network. 
Ramaswami's research provides a meaningful study of the RWA problem at that time, and has been significantly referenced by subsequent studies [19-22].

In 2005, X. Yang et al. published research based on translucent optical networks (optical network implemented with regeneration nodes) [23]. They propose heuristic algorithms to optimize the allocation of regeneration nodes while solving the RWA problem. Yang's work is meaningful for subsequent studies on translucent optical networks [24-26].

In 2010, K. Christodoulopoulos et al. published research on the static resource allocation problem in EONs [27]. That paper considers the RSA problem for EON and analyzes several heuristic algorithms, testing the performance of each algorithm. While Christodoulopoulos's research had a great influence on MILP formulations and future studies on the RSA problem, his paper only considers the basic RSA problem without PLIs.

In 2014, X. Wang et al. solved the RSA problem in a large scale EON (NSF24) with a fast heuristic algorithm, referred to as the recursive MILP (re-MILP), for solving the RSA problem in a short time [2]. Wang's work also introduces the allocation of regeneration nodes and their impact on RSA. Wang's work guarantees the QoT using the TR model. However, in addition to the fact that the TR model overestimates the signal to interference plus noise ratio (SINR) condition in EONs, the parameters of the TR model used in Wang's work were based on samples acquired through laboratory results [13]. Thus, Wang's TR model is not universally applicable. The performance of Wang's re-MILP, compared with the optimal solution, has the potential to be improved.

In 2015, J. Zhao et al. published research on resource provision algorithms in EON suffering from PLIs [4]. The authors use the standard GN model with a lookup table to translate the nonlinear standard GN model into a linear model. They model 
the RSA as an ILP problem. However, this algorithm is extremely time consuming and only applicable to a limited number of demands in small network topologies.

In 2015, L. Yan et al. published research on RSA problem in flexible grid networks with the impacts of PLIs. The authors use an MILP model with a finely linearized GN model applied to flexible grid networks [6,12]. However, because of this finely linearized GN model, the process of Yan's RSA problem is again time-consuming because of the massive computation resources required.

In 2016, M. Klinkowski et al. published research on the routing, spectrum, transceiver and regeneration allocation (RSTRA) problem that is an extension of the conventional RSA problem [28]. In order to efficiently address the RSTRA problem in EONs, the authors propose a heuristic algorithm, referred to as the minimum cost light-paths assignment for ordered demands algorithm. They use a simplified transmission reach model to ensure the QoT, resulting in over-provisioning, and thus unnecessary costs.

\subsection{Thesis Outline}

This thesis is organized as follows. In Chapter 2, we introduce some terms, background knowledge and definitions used in the research. In Chapter 3, we describe the proposed models: the conservative linearized Gaussian noise (CLGN) model and a novel transmission reach model, the Gaussian-noise-based transmission reach (GNTR) model. In Chapter 4, we then elaborate on the MILP model of the RSA problem and our heuristic algorithm, referred to as the sequential allocation algorithm. Chapter 5 provides numerical results and analysis based on simulation. In Chapter 6, we draw conclusions and list opportunities for future work. 


\section{Chapter 2}

\section{EON Description and Problem Formulation}

In order to completely understand the RSA problem for EONs suffering from PLIs, we introduce the fundamental concepts of EONs and two kinds of analytical models for ensuring the QoT requirements in Sections 2.1-2.3. Furthermore, signal regeneration, as a modern technique to enhance the performance of EONs, is explained in Section 2.4. Finally, in Sections 2.5-2.6, we introduce the overall picture of the RSA problem and heuristic algorithms.

\subsection{Elastic Optical Network}

EONs exhibit great potential in regard to being highly efficient and flexible, which saves network resources. EONs are able to support both low transmission rates and high transmission rates simultaneously [8]. EONs are able to choose a modulation format for each demand that satisfies the QoT requirements through transmission with minimal spectrum usage. However, in conventional DWDM networks, the optical transmission reach, the channel bit rate, and the optical spectrum are fixed [1]. 
However, some literature $[8,29]$ considers that full elasticity, i.e., an infinitely small granularity of the sub-carriers, might not be easily accomplished by current techniques. Therefore, less-elastic optical networks, referred to as flexible grid networks, have been proposed as a more realistic version of EONs. [8]. Flexible grid networks have a granularity of $12.5 \mathrm{GHz}$, dividing the spectrum into specific nonoverlapping slots. Although the flexibility of the flexible grid network with $12.5 \mathrm{GHz}$ granularity is better than the ITU DWDM with a $50 \mathrm{GHz}$ grid, there is still finite granularity in the network. Through further development of techniques such as more advanced flexible bandwidth transmitters and receivers, the full elasticity of the network can successfully be achieved. In addition, the flexible grid optical network can be considered as a special case of an EON. To make this research more general, this thesis focuses on general EONs instead of flexible grid networks.

In summary, there are two main properties of EONs. First, the light-path can be generated with heterogeneous bit rates. Second, the BVT can generate an arbitrary spectrum. These two properties of EONs enable the high efficiency and the flexibility [1]. Because of these properties and the merits of EONs, proper planning for EONs could bring enormous benefits. However, the PLIs are unavoidable in large EONs, especially when we consider that a great number of demands are transmitted in backbone networks [30]. The PLIs affect the channel quality and therefore the quality of the received signal. Estimation of PLIs in EONs is important in the network planning stage (designing networks and planning usage of network resources) because using conservative estimates leads to resource over-provisioning. 


\subsection{Gaussian Noise Model and Quality of Trans- mission}

There are several main types of PLIs: nonlinear noise, chromatic dispersion and amplified spontaneous emission (ASE) noise. Since the chromatic dispersion can be compensated by digital signal processing, we only need to consider the impairments caused by the nonlinear interference (NLI) (caused by the interaction of nonlinearity and dispersion) and the ASE noise (caused by the Erbium-doped fiber amplifiers (EDFAs)). Hence, the NLI and the ASE are important when estimating the QoT $[12]$.

The fiber loss in an optical network is usually $0.2 \mathrm{~dB} / \mathrm{km}$. Each span, i.e., the length between two EDFAs, is usually $100 \mathrm{~km}$. The transmitted power is attenuated by $20 \mathrm{~dB}$ at the end of each span [5]. The photo-detector at the receiver is unable to detect the signal with sufficient quality, leading to the necessity of using EDFAs as signal amplifiers at the end of each span $[5,31]$. However, the amplifying process will cause ASE noise, which is modeled as additive Gaussian noise with power spectral density (PSD) given as [5]

$$
G_{A S E}^{s p a n}=\left(e^{\alpha L}-1\right) \hbar \nu n_{s p}
$$

where $n_{s p}$ represents the spontaneous emission factor, $\hbar$ represents Planck's constant, $\alpha$ represents the fiber power attenuation, and $L$ represents the fiber length per span. Note that we assume the gain of the EDFAs is frequency flat [6] and an EDFA exactly compensates the span loss [5].

The GN model used for analytically estimating the NLI PSD is valid based on several assumptions, as stated in [5,6]: The fiber links are dispersion uncompensated 
fibers (i.e. the fiber link is purely compensated by digital signal processing) with enough length. The signal PSD is homogeneous for each polarization. The fiber loss and chromatic dispersion are totally compensated and negated. The NLI PSD is accumulated along the light path. The affecting channels are non-overlapping in spectrum.

With the above assumptions, the GN model can be applied to estimate the signal QoT. The NLI effects can be divided into self channel interference (SCI) and cross channel interference (XCI) [6,32]:

$$
G_{N L I, i}^{s p a n}=G_{S C I, i}^{s p a n}+G_{X C I, i}^{s p a n} .
$$

where $G_{N L I, i}^{s p a n}$ represents the $i$ th channel's NLI PSD per span, $G_{S C I, i}^{s p a n}$ represents the $i$ th channel's SCI PSD per span, and $G_{X C I, i}^{s p a n}$ represents the $i$ th channel's XCI PSD per span [5]. SCI is caused by the channel itself, only varying with the bandwidth of that channel $[6,32]$ :

$$
G_{S C I, i}^{\text {span }}=\mu G i\left(G_{i}^{2} \operatorname{arcsinh}\left(\rho \Delta f_{i}^{2}\right)\right)
$$

where $\rho=\left(\pi^{2}\left|\beta_{2}\right|\right) / 2 \alpha, \mu=\left(3 \gamma^{2}\right) /\left(2 \pi \alpha\left|\beta_{2}\right|\right), \gamma$ represents the fiber nonlinear parameter, $\beta_{2}$ represents the group velocity dispersion parameter, $\Delta f_{i}$ represents the $i$ th channel's bandwidth, and $G_{i}$ represents the $i$ th channel's signal PSD. When $\Delta f_{i}$ is large, the inverse hyperbolic sine function and the logarithm function are similar $[6,32]$. Equation (2.3) can thus be replaced by [15]

$$
G_{S C I, i}^{\text {span }}=\mu G i\left(G_{i}^{2} \ln \left(\rho \Delta f_{i}^{2}\right)\right) .
$$

The XCI is caused by the interaction between channels. It depends on the difference 
in center frequencies and bandwidths of the affecting channels $[6,32]$ :

$$
G_{X C I, i}^{s p a n}=\mu G i\left(G_{j}^{2} \sum_{j=1 ; j \neq i}^{M_{c}} \ln \left(\frac{|f i-f j|+\Delta f_{j} / 2}{|f i-f j|-\Delta f_{j} / 2}\right)\right)
$$

where $M_{c}$ represents the total number of channels on the same fiber link as the $i$ th channel and $f_{k}$ represents the $k$ th channel's center frequency.

The QoT for each channel at the receiver side is the bit error rate (BER), which is related to the SINR, given the modulation format. This thesis focuses on the BER before the forward error correction (FEC) process, referred to as the pre-FEC BER. The pre-FEC BER used in this thesis is $4 \times 10^{-3}$ [5]. In order to guarantee the desired QoT, which is measured by the pre-FEC BER, the actual SINR over each transparent segment (light-path segment without signal regeneration) must satisfy the threshold SINR [5]:

$$
\mathrm{SINR}_{i} \geq \mathrm{SINR}_{i}^{t h}
$$

where the $\mathrm{SINR}_{i}$ is the actual signal to interference plus noise ratio for the $i$ th channel and $\operatorname{SINR}_{i}^{\text {th }}$ is the threshold SINR (the minimum SINR satisfying the QoT requirements) for the $i$ th channel [5]. Hence, the SINR constraint (2.6) becomes:

$$
\mathrm{SINR}_{i}=\frac{G_{i}}{\left(G_{N L I, i}^{\text {span }}+G_{A S E, i}^{\text {span }}\right) N_{s}} \geq \mathrm{SINR}_{i}^{t h}
$$

where $N_{s}$ represents the number of spans on the transparent segment. For common modulation formats, values for threshold SINR are listed in Table 2.1. 
Table 2.1: Modulation Format, Spectral Efficiency and Threshold SINR $\left(\right.$ pre-FEC BER $\left.=4 \times 10^{-3}\right)[5]$

\begin{tabular}{c|c|c}
\hline Modulation Format & spectral efficiency $\eta(\mathrm{bit} / \mathrm{s} / \mathrm{Hz})$ & $\mathrm{SINR}_{i}^{\text {th }}$ \\
\hline PM-BPSK $^{1}$ & 2 & 3.52 \\
PM-QPSK $^{2}$ & 4 & 7.03 \\
\hline
\end{tabular}

\subsection{Transmission Reach Model}

As a simpler alternative to the GN model, the TR model is broadly used for estimating PLIs to ensure the QoT is met in long-haul transmission systems. The TR model is applied in most research addressing the RSA problem because of its simplicity [16]. Additionally, the TR model is linear, so it can easily be implemented in linear programming algorithms.

The TR model estimates the longest transparent segment length a signal can travel and still satisfy a conservative estimate of the SINR. The disadvantage of the TR model is that it does not take the instantaneous channel state into account. Moreover, the parameters of the TR model applied by some researchers are obtained from experimental results [13]. These experimental results are drawn based on different experimental setups, thus lead to questions on the universality of these results. Additionally, the laboratory results are discrete values instead of a continuous function, resulting in model inaccuracies [2]. Instead of implementing the TR model based on experimental data, we implement a GN model based analytic algorithm to generate the parameters of the TR model in order to make the comparison with the

\footnotetext{
${ }^{1}$ PM-BPSK: polarization-multiplexed binary phase-shift keying

${ }^{2}$ PM-QPSK: polarization-multiplexed quadrature phase-shift keying
} 
CLGN model fair. In general, because of the state-independence of the TR model, using this model in the network planning stage leads to resource over-provisioning and unnecessary costs.

\subsection{Signal Regeneration}

Because the accumulated PLIs constantly harm the systems, the transmitted signal may not satisfy the desired QoT. Consequently, detecting the transmitted signal and recovering the original information may fail at the receiver side. Hence, regeneration nodes that perform optical-electrical-optical (OEO) conversion for reducing the impairments are needed as intermediate nodes [31]. The regeneration (including re-timing, re-shaping and re-amplification) is an electrical process functioning at the intermediate nodes. We assume the PLIs are fully negated through the regeneration process [2].

A plan for allocation of regeneration nodes should account for the high cost of highspeed electronic equipment. This equipment's high cost necessarily implies a similar cost for OEO conversion. These considerations require a careful and conservative aforementioned allocation plan [2]. Because one regeneration circuit can only serve one signal, and a limited number of regeneration circuits per regeneration node is assumed, not all signals can be regenerated at a regeneration nodes. And again, the appropriate allocation of regeneration nodes could bring significant benefits. 


\subsection{Routing and Spectrum Allocation (RSA) Prob- lem}

Routing and wavelength allocation (RWA) algorithms are proposed to coordinate the wavelength routing and the assignment simultaneously in order to obtain the best solution for light-path deployment in fixed grid DWDM networks with $50 \mathrm{GHz}$ frequency spacing [33]. In the conventional RWA problem, routing and wavelength assignment for demands are optimized to obtain the minimum resource usage.

The RSA problem in EONs is an analog of the RWA problem in DWDM networks [34]. Unlike the RWA problem, the demands in the RSA problem may be deployed with various transmission rate requirements and modulation schemes [5]. In the RWA problem, a demand is transmitted in a $50 \mathrm{GHz}$ frequency slot with a fixed discrete center frequency $[6,12]$. However, in EONs, the $50 \mathrm{GHz}$ frequency slot is further divided into infinitely many narrow frequency slots. Therefore, in the RSA problem, a demand is transmitted in a flexible spectrum (a number of narrow frequency slots) from its source to its destination [1,29,34].

In EONs, without the constraints of a fixed grid in the network, the frequency slots, also known as the spectrum, can be assigned seamlessly. The RSA problem in EONs is to appropriately route the path of the demands and to carefully assign the required spectrum for the demands, in order to save network resources. Since a demand can be assigned a modulation format that provides desired performance, selection of the modulation formats for each demand along its light-path affects the resources needed by the EONs.

Moreover, when regeneration is considered, the noise accumulated along the lightpath is reduced after the OEO conversion process. Hence, with the implementation 
of regeneration nodes, constraints based on either the TR or the GN models are able to guarantee that all demands satisfy the QoT for practical networks.

\subsection{Heuristic Method}

Heuristic algorithms are used for solving optimization problems to achieve a tradeoff between the complexity of the problems and a guarantee of optimality. RSA problems are NP-hard [35], usually formulated as MILPs. An MILP is an algorithm to realize the best outcome in a mathematical model with linear constraints and objective function. Some variables in MILP are integers, whereas other variables are non-integers [36]. Unlike heuristic algorithms, MILPs are able to provide the optimal solution. However, due to the existence of integer variables, which come from the decision variables in the RSA problems, MILP solvers must spend a significant amount of time determining the integer variables. Therefore, optimal solutions are not able to be obtained within a reasonable time using MILPs. Especially with large problem dimensions, obtaining the optimal solutions requires astronomically high computation resources [2].

However, heuristic algorithms are proposed to solve optimization problems within a reasonable time and obtain near-optimal solutions. Because of the high scalability as well as the lower computational resources required, heuristic algorithms [31] have been broadly applied $[2,17,27,37,38]$. [37] accommodates demands in accordance with the length of the routing paths in order to appropriately coordinate the network resources usage while speeding up the solving process. [27] proposes a heuristic algorithm, referred to as the $\mathrm{R}+\mathrm{SA}$ algorithm, which decomposes the RSA problem into two sub-problems (a routing problem and a spectrum allocation problem). After solving the routing problem, the $\mathrm{R}+\mathrm{SA}$ algorithm then assigns spectrum to these routed 
light-paths. Heuristic algorithms are efficient sub-optimal algorithms for solving the RSA problem [31]. However, when the complexity of the problem increases, the entire variable space is not explored within a permitted time period, leading to non-ideal performance of these algorithms $[2,12,31,39]$. 


\section{Chapter 3}

\section{Conservative Linearized GN Model}

In this chapter, we introduce the CLGN model in Section 3.1. In Section 3.2, we introduce the GN model based TR model, referred to as the GNTR model. In Section 3.3, we simulate the GN model, the CLGN model, and the GNTR model in order to analyze their link level performance.

\subsection{Conservative Linearized Gaussian Noise (CLGN) Model}

In order to be processed by the MILP engines, we propose a linearized version of the standard GN model. The principles for linearizing the standard GN model are listed as follows. First, the linearized GN model cannot exceed the QoT estimation of the standard GN model. Second, the linearized GN model should have similar QoT estimation for the most realistic cases. Third, the linearized GN model needs to be linear in the variables used by the MILP.

For the RSA problem, the bandwidth of demands $\left(\Delta f_{i}, \Delta f_{j}\right)$ are given as optimization inputs. However, the total number of demands on the fiber link $\left(M_{c}\right)$ and 
the center frequencies of demands $\left(f_{i}, f_{j}\right)$ are decision variables in this optimization problem. Therefore, the SCI term is a linear function of the MILP variables in the standard GN model equation (2.3). The term that needs to be linearized is the XCI term. Since the variable $\left|f_{i}-f_{j}\right|$ is inside a logarithm function, we consider an upper bound on the XCI term as

$$
\begin{aligned}
G_{N L I, i}^{\text {span }} & =\mu G i\left(G_{i}^{2} \ln \left(\rho \Delta f_{i}^{2}\right)+\sum_{j=1 ; j \neq i}^{M_{c}} G_{j}^{2} \ln \left(\frac{|f i-f j|+\Delta f_{j} / 2}{|f i-f j|-\Delta f_{j} / 2}\right)\right), \\
& =\mu G_{i}\left(G_{i}^{2} \ln \left(\rho \Delta f_{i}^{2}\right)+\sum_{j=1 ; j \neq i}^{M_{c}} G_{j}^{2} \ln \left(\frac{\Delta f_{j}}{|f i-f j|-\Delta f_{j} / 2}+1\right)\right), \\
& \leq \mu G_{i}\left(G_{i}^{2} \ln \left(\rho \Delta f_{i}^{2}\right)+\sum_{j=1 ; j \neq i}^{M_{c}} G_{j}^{2} \ln \left(\frac{\Delta f_{j}}{\Delta_{g b}+\Delta f_{j} / 2}+1\right)\right)
\end{aligned}
$$

where $G_{N L I, i}^{s p a n}$ is the $i$ th channel's NLI PSD per span, and $\Delta_{g b}$ is the guard band. We refer to this linearized version of the standard GN model as the conservative linearized Gaussian noise (CLGN) model.

From a spectrum perspective, for the CLGN model we consider that all connections $j$ that contribute to the XCI for demand $i$ are located as close as possible to the demand (without considering the actual center frequency difference $\left|f_{i}-f_{j}\right|$ ). The CLGN model is a conservative XCI estimation. When there are a large number of demands deployed on the same fiber link, the CLGN model provides an overestimated XCI compared with the standard GN model. On the other hand, when there are few demands on the fiber link, the CLGN model is able to provide a similar XCI estimate compared with the standard GN model. 


\subsection{Gaussian Noise Based Transmission Reach (GNTR) Model}

In order to quantify the benefits of the CLGN model, we wish to compare the CLGN model with the TR model, a model that is broadly applied in published research. However, the disadvantages of the existing TR model are listed below. Instead of imposing a constraint on the QoT as the CLGN model does, the TR model imposes a constraint over the transparent transmission distance. Most existing TR models applied in published research are based on experimental data. Different experimental setups (fiber parameters, experimental circumstances, signal PSD and QoT requirements) result in different experimental results. Hence, the TR parameters based on existing experimental data remain problematic when comparing with the CLGN model. Furthermore, the experimental data values are discrete values instead of a continuous function of transmission distance. Hence, we propose a GN-based analytic algorithm to generate the parameters for a TR model in order to make the comparison with the CLGN model fair.

The GN-based transmission reach (GNTR) is the shortest transmission reach based on the standard GN model given the bandwidth of a demand, input PSD, and QoT requirements. In order to obtain this TR, we first consider the worst case noise level: $N_{G N T R}=\max _{M_{c}, \Delta f_{j}}\left(G_{N L I, i}^{\text {span }}+G_{A S E, i}^{\text {span }}\right)$, where $N_{G N T R}$ denotes the worst case noise for the GNTR model, and $\max _{M_{c}, \Delta f_{j}}\left(G_{N L I, i}^{\text {span }}+G_{A S E, i}^{\text {span }}\right)$ denotes the process of obtaining the worst case noise level over $M_{c}$ and $\Delta f_{j}$. After obtaining the worst case noise level, the GNTR can be calculated as

$$
T_{i}^{G N T R}=\frac{G_{i}}{\operatorname{SINR}_{i}^{t h} \max _{M_{c}, \Delta f_{j}}\left(G_{N L I, i}^{\text {span }}+G_{A S E, i}^{\text {span }}\right)} \times L,
$$






Figure 3.1: Illustration of interfering demands positioned in the optical spectrum.

where $T_{i}^{G N T R}$ is the GNTR of the $i$ th channel.

In (3.1), given $\Delta f_{i}\left(f_{i}\right.$ is at the center of the spectrum), the input PSD, and the fiber parameters, the XCI term is the only part that can vary. The XCI term depends on $M_{c}$ and $\Delta_{j}$. Therefore, as shown in Figure 3.1, we vary $M_{c}$ and $\Delta_{j}$ (fully occupying the $4000 \mathrm{GHz}$ spectrum [40]) in order to obtain the worst case noise:

$$
\begin{gathered}
\arg \max _{M_{c}, \Delta f_{j}} G_{X C I, i}^{\text {span }}=\mu G i\left(G_{j}^{2} \sum_{j=1 ; j \neq i}^{M_{c}} \ln \left(\frac{|f i-f j|+\Delta f_{j} / 2}{|f i-f j|-\Delta f_{j} / 2}\right)\right), \\
\text { s.t. } \quad \Delta_{g b} \times\left(M_{c}-1\right)+\Delta f_{i}+\sum_{j=1}^{M_{c}-1} \Delta f_{j}=4000,
\end{gathered}
$$

where the fiber parameters are listed in Table 3.1. We assume the input PSD for each channel is the same, denoted as $G$.

Based on Figure 3.2, we can obtain the GNTR based on the worst case noise level calculated by the standard GN model, given the threshold SINR and modulation format. As shown in Figure 3.3, the worst transmission reach occurs when a large demand is allocated on each side of the test demand $i$, as proved in $[41,42]$.

After comparing the GNTR model with the TR model based on experimental data [2], our algorithm reveals fairness, because the GNTR and CLGN models are GN model based analytic algorithms. The GNTR algorithm can serve demands with an arbitrary bandwidth. Moreover, our GNTR is independent of experimental results 
Table 3.1: Fiber Parameters [6]

\begin{tabular}{l|l}
\hline \hline$\Delta_{g b}$ & spectral guard band: $12.5 \mathrm{GHz} ;$ \\
$G$ & input signal PSD: $0.015 \mathrm{~W} / \mathrm{THz}$ \\
$\alpha$ & attenuation of fiber: $0.22 \mathrm{~dB} / \mathrm{km}$ \\
$\hbar$ & Planck's constant \\
$n_{s p}$ & spontaneous emission factor: 1.58 \\
$\gamma$ & fiber nonlinearity coefficient: $1.32 \times 10^{-3}(W \cdot m)^{-1}$ \\
$\beta_{2}$ & fiber group velocity dispersion parameter: $-21.7 \mathrm{ps}^{2} / \mathrm{km}^{-1}$ \\
$L$ & fiber length of per span: $100 \mathrm{~km}$ \\
$\nu$ & optical carrier frequency: $193.55 \mathrm{THz}$ \\
$\rho$ & $\rho=\left(\pi^{2}\left|\beta_{2}\right|\right) / 2 \alpha$ \\
$\mu$ & $\mu=\left(3 \gamma^{2}\right) /\left(2 \pi \alpha\left|\beta_{2}\right|\right)$ \\
\hline \hline
\end{tabular}




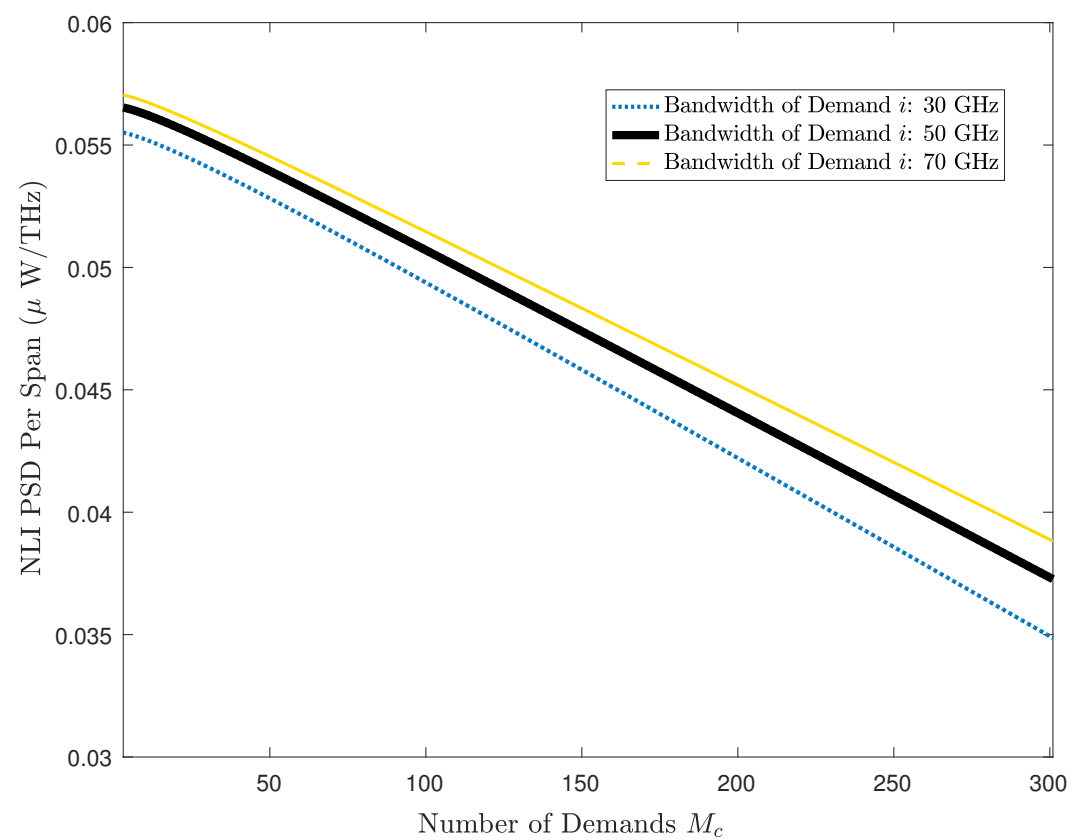

Figure 3.2: NLI PSD per span versus the number of demands shared on the same fiber link, $M_{c}$, filling the $4000 \mathrm{GHz}$ spectrum.



Figure 3.3: Illustration of the worst case interference on test demand $i$. 
and therefore does not require interpolation. Our proposed algorithm is able to obtain the transmission reach for systems that have not been tested experimentally. In this thesis, the TR model we implement for simulation is based on the GNTR algorithm.

\subsection{Link Level Analysis}

We simulate the standard GN model, the CLGN model and the GNTR model in order to analyze the link level performance of each estimation model of PLIs, for various QoT requirements.

In this thesis, we define $N_{C L G N}=\mathbf{C L}\left(G_{N L I, i}^{s p a n}+G_{A S E, i}^{s p a n}\right), N_{G N T R}=\max \left(G_{N L I, i}^{s p a n}+\right.$ $\left.G_{A S E, i}^{s p a n}\right)$, and $N_{G N}=\left(G_{N L I, i}^{s p a n}+G_{A S E, i}^{s p a n}\right)$, where $N_{C L G N}$ is the noise level estimated by the CLGN model. CL represents the conservatively linearizing process of the GN model:

$$
\begin{aligned}
\mathbf{C L}\left(G_{N L I, i}^{\text {span }}+G_{A S E, i}^{\text {span }}\right) \doteq & \mu G_{i}\left(G_{i}^{2} \ln \left(\rho \Delta f_{i}^{2}\right)+\sum_{j=1 ; j \neq i}^{M_{c}} G_{j}^{2} \ln \left(\frac{\Delta f_{j}}{\Delta_{g b}+\Delta f_{j} / 2}+1\right)\right) \\
& +\left(e^{\alpha L}-1\right) \hbar \nu n_{s p} .
\end{aligned}
$$

$N_{G N T R}$ is the noise level estimated by the GNTR model, and $N_{G N}$ is the noise level estimated by the standard GN model. In all our analysis we assume the GN model yields an accurate approximation to the PLIs.

We consider two simulation scenarios to compare the various PLI models. In the first scenario, there are several equal-bandwidth demands deployed on the same fiber link: $\Delta f_{i}=\Delta f_{j}$ for $j=1,2, \ldots, M_{c}$ (demand $i$ is at the center of the spectrum). We simulate four cases separately: $M_{c}=3,5,7,9$. We then obtain the GNTR, the TR based on the CLGN model, and the TR based on the standard GN model for each $M_{c}$. The GNTR $\left(T_{i}^{G N T R}\right)$ is shown in (3.4). The TR of the CLGN model $\left(T_{i}^{\mathrm{CLGN}}\right)$ is 


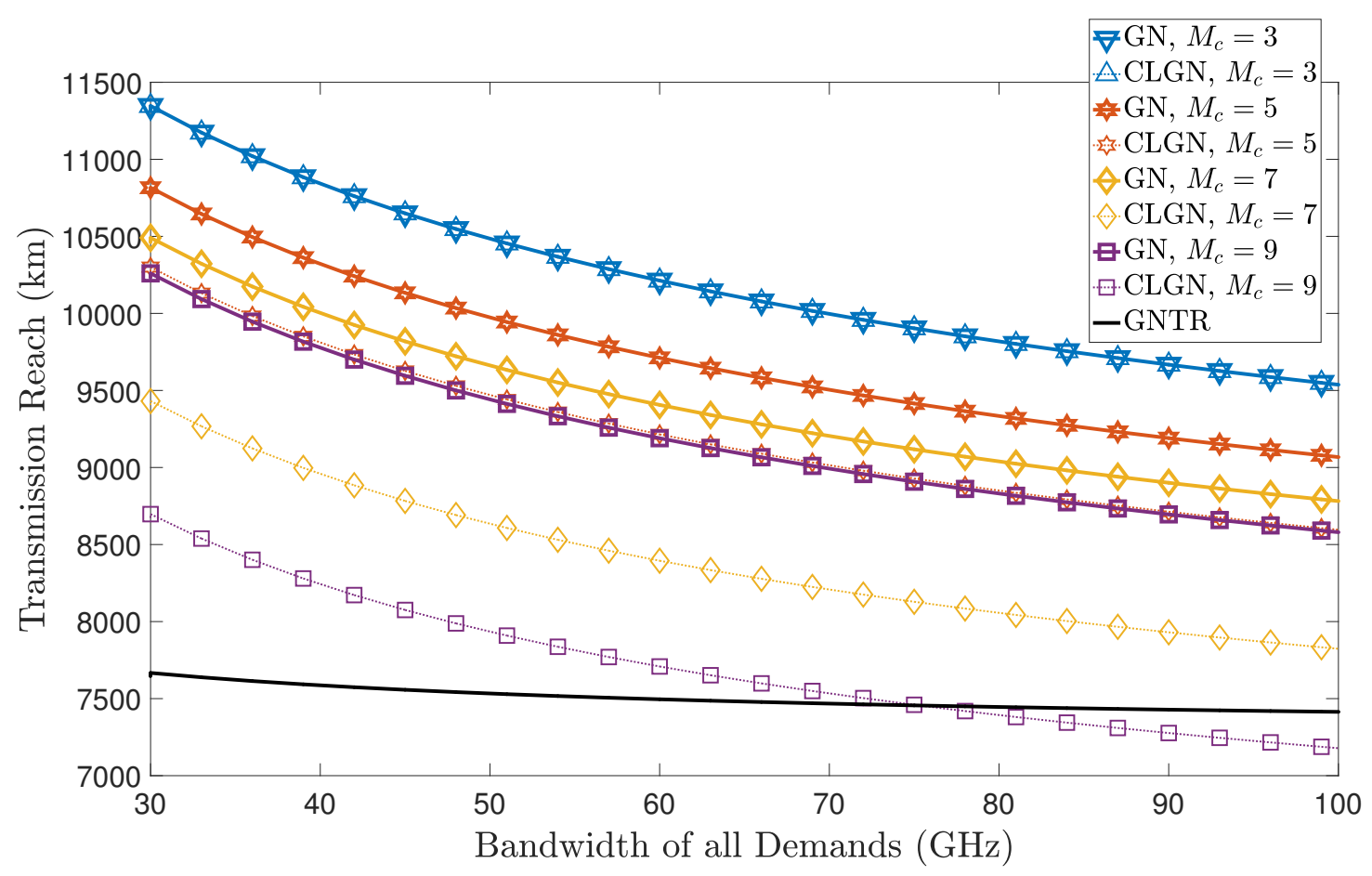

Figure 3.4: Comparison of the transmission reach generated by the GNTR, the CLGN, and the GN models, for various $M_{c}$, with BPSK modulation.

obtained by

$$
T_{i}^{\mathrm{CLGN}}=\frac{G_{\text {input }, i}}{\operatorname{SINR}_{i}^{t h} N_{C L G N}} \times L .
$$

The TR of the standard GN model $\left(T_{i}^{\mathrm{GN}}\right)$ is obtained by

$$
T_{i}^{\mathrm{GN}}=\frac{G_{\text {input }, i}}{\operatorname{SINR}_{i}^{t h} N_{G N}} \times L
$$

Figures 3.4 and 3.5 show that for both QPSK and BPSK modulation formats, the CLGN model provides a better estimate of the true TR compared with the GNTR model. When $M_{c}$ increases, the gaps between the TR based on the CLGN model and the standard GN model increases. When $M_{c}=9$ and the bandwidth of each demand exceeds $78 \mathrm{GHz}$, the GNTR model outperforms the CLGN model in providing 


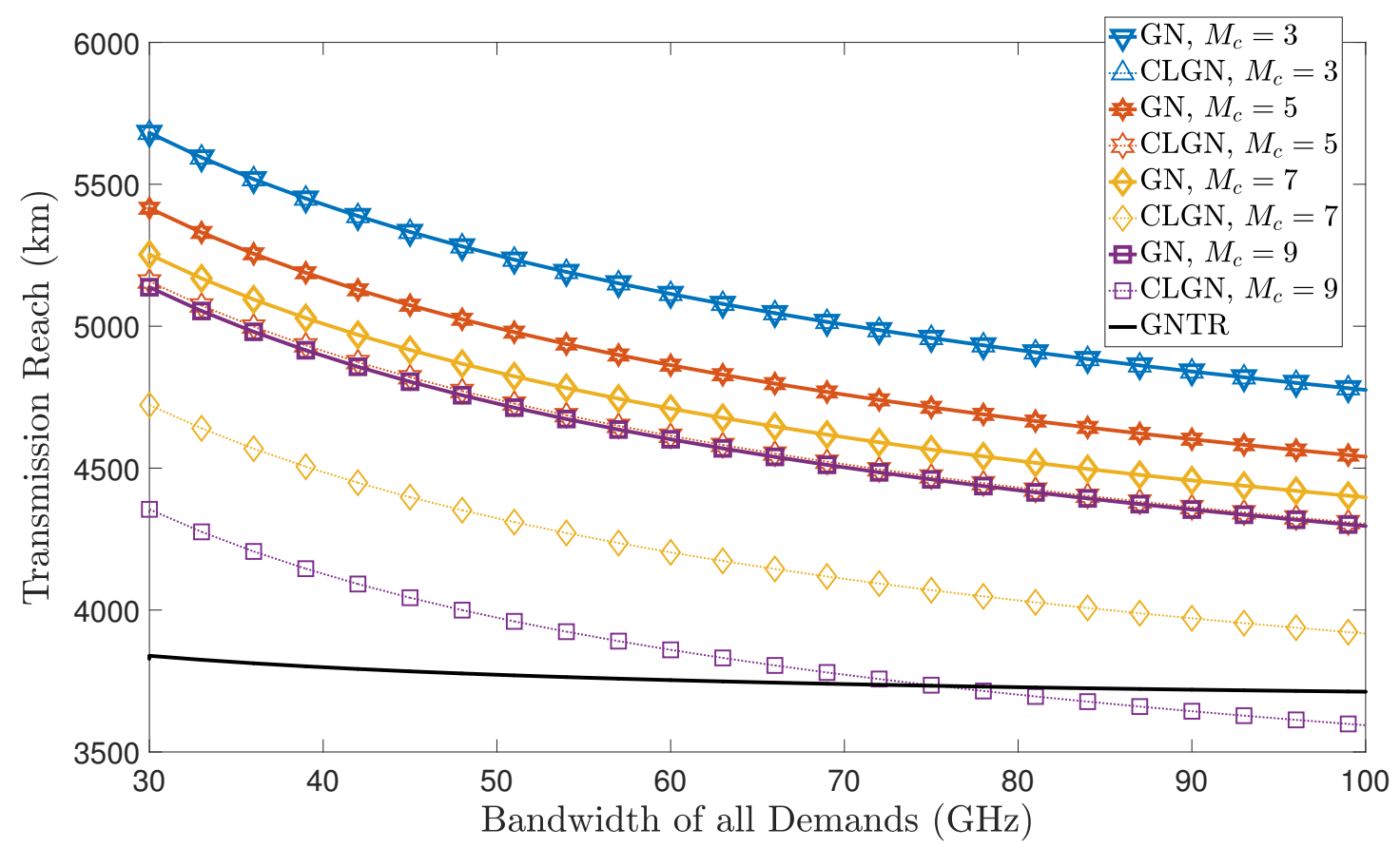

Figure 3.5: Comparison of the transmission reach generated by the GNTR, the CLGN, and the GN models, for various $M_{c}$, with QPSK modulation.

an estimate of the TR, because the CLGN model is a conservative approximation. However, the GNTR model curve does not depend on $M_{c}$.

We compute the normalized link noise estimation error, using the standard GN model as a reference, as

$$
\operatorname{Err}_{*}=\frac{\left|N_{*}-N_{G N}\right|}{N_{G N}}, \quad *=C L G N, G N T R
$$

Figure 3.6 shows the link noise estimation error comparison between the GNTR model and the CLGN model. The estimation error of the CLGN model is always smaller than that of the GNTR model when $M_{c} \leq 7$. When $M_{c}=9$ and the bandwidth of each demand exceeds $78 \mathrm{GHz}$, the estimation error of the CLGN model is worse than that of the GNTR model. In general, we can conclude that the CLGN model has a better estimation accuracy than the GNTR model. In addition, the 


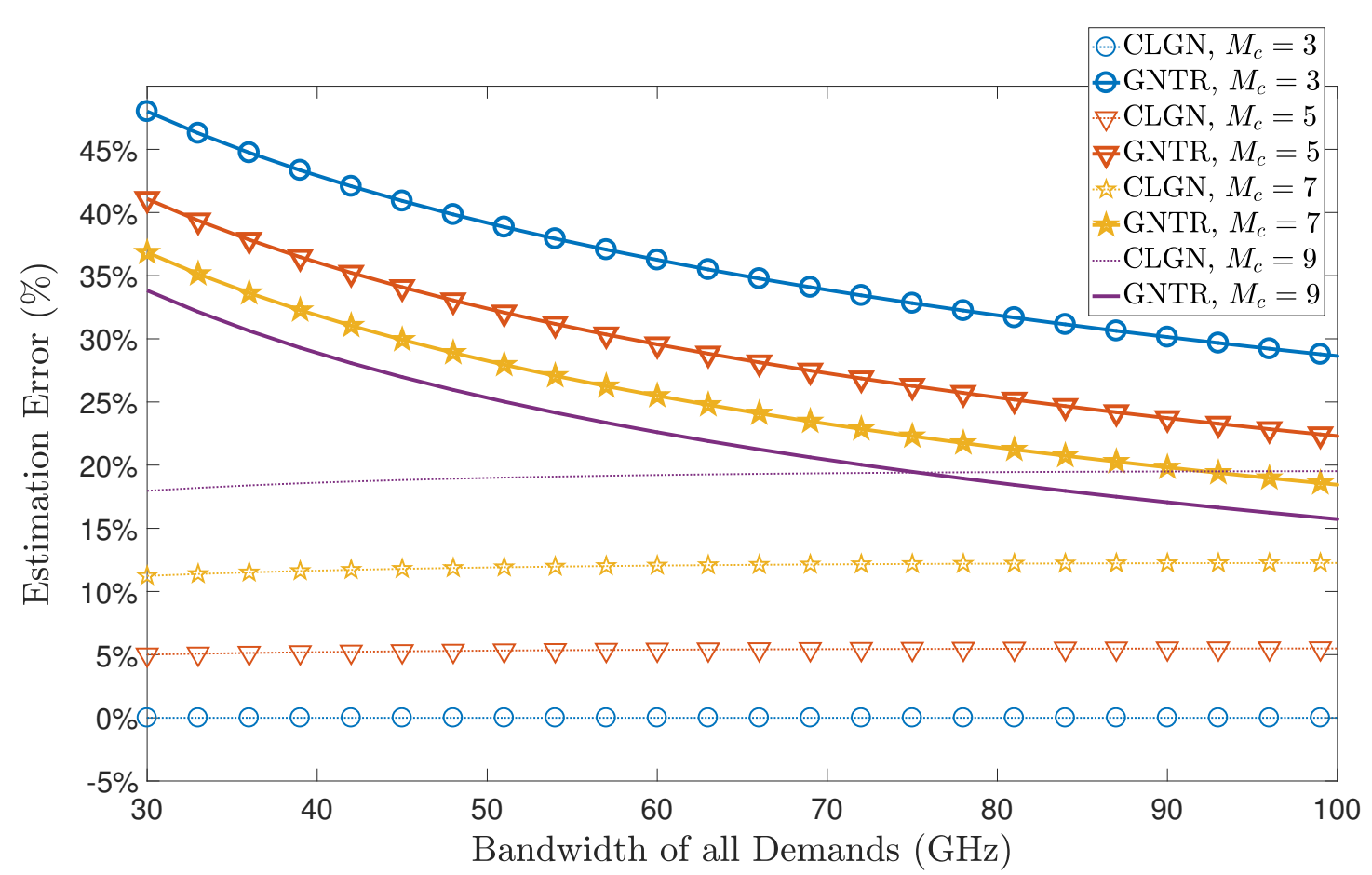

Figure 3.6: Comparison of the estimation error $\left(\operatorname{Err}_{*}\right)$ generated by the GNTR, the CLGN, and the GN models, for various $M_{c}$.

estimation accuracy of the CLGN model decreases as $M_{c}$ increases, as expected.

In the second scenario, we simulate several demands with random bandwidths deployed on the same fiber link. A probabilistic analysis is implemented in this scenario for comparing the performance of the standard GN model, the CLGN model, and the GNTR model. Each demand has a bandwidth uniformly distributed from 30 to $100 \mathrm{GHz}, \Delta f_{i}, \Delta f_{j} \sim U[30,100]$. After completing 10000 simulation trials for different values of $M_{c}\left(M_{c}=3,5,7\right.$, and 9$)$, we estimate the probability distribution of both normalized (count or frequency of observations [43]) noise level $\left(N_{G N}, N_{G N T R}\right.$, and $\left.N_{C L G N}\right)$ and the transmission reach $\left(T_{i}^{\mathrm{GN}}, T_{i}^{\mathrm{GNTR}}\right.$, and $\left.T_{i}^{\mathrm{CLGN}}\right)$ using histograms.

In Figures 3.7 and 3.9, we show that the CLGN model is better at estimating the performance of the PLIs than the GNTR model for $M_{c} \leq 7$, assuming the GN model yields an accurate approximation to the PLIs. When $M_{c}=9$, the CLGN model has a greater than $60 \%$ probability of having a better performance than the 

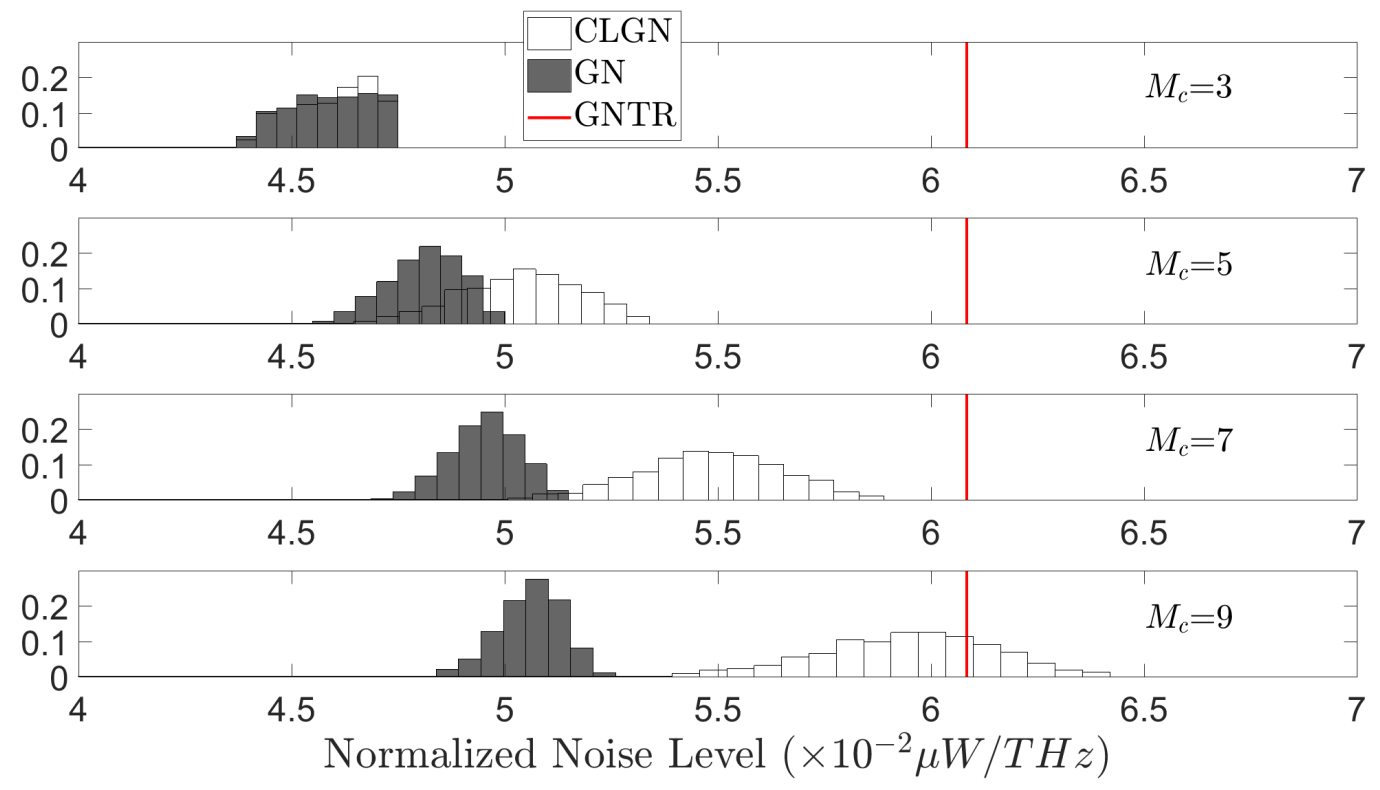

Figure 3.7: Histogram of normalized noise level in BPSK with various $M_{c}$.
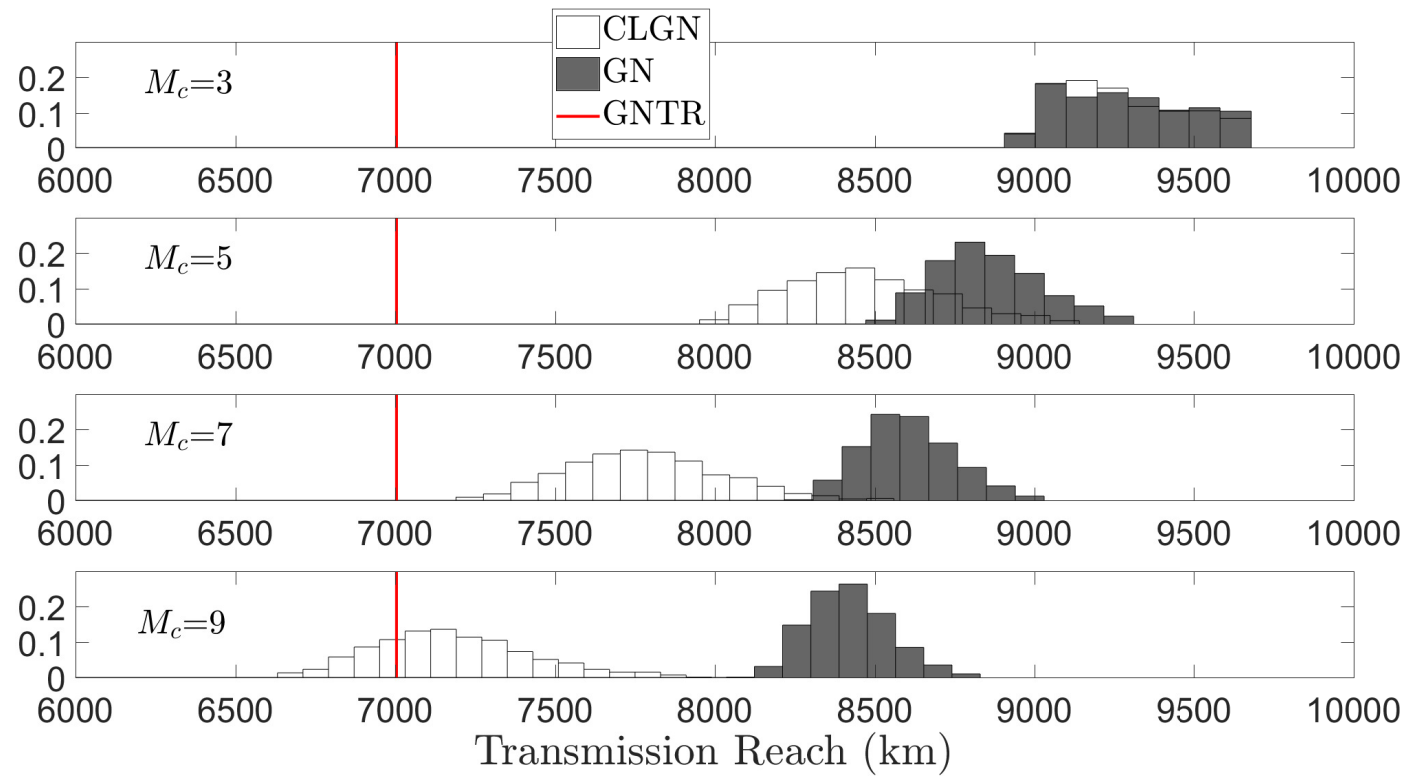

Figure 3.8: Histogram of transmission reach in BPSK with various $M_{c}$. 

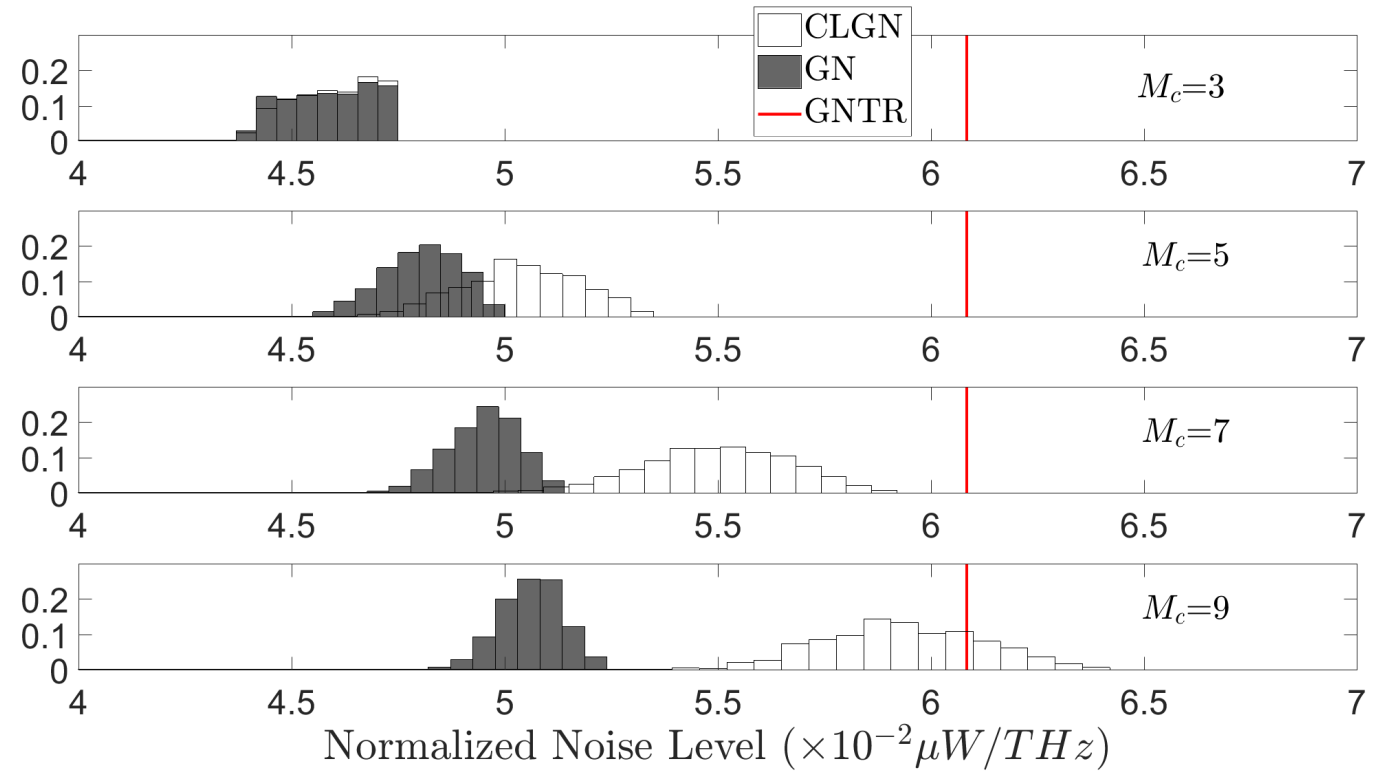

Figure 3.9: Histogram of normalized noise level in QPSK with various $M_{c}$.


Figure 3.10: Histogram of transmission reach in QPSK with various $M_{c}$. 
GNTR model. In Figures 3.8 and 3.10, the CLGN model estimates the TR better than the GNTR model, when $M_{c}<9$. When $M_{c}=9$, the CLGN model has a greater than $60 \%$ probability of outperforming the GNTR model in transmission reach. In this simulation scenario, the simulation settings are similar to settings used in [2], [3], and [4]. Hence, based on these link level analyses, we can conclude that the CLGN model has better performance in estimating PLIs than the GNTR model for many cases of practical interest. 


\section{Chapter 4}

\section{Heuristic Algorithm for RSA in EONs}

In this chapter, we introduce the MILP model for the RSA problem and our proposed heuristic algorithm. In Sections 4.1-4.2, we introduce a basic MILP formulation for addressing the all-optical RSA problem without considering PLIs. In Sections 4.34.4, we extend the basic MILP with consideration of QoT requirements and signal regeneration. In Sections 4.5-4.6, we propose a heuristic algorithm, referred to as the sequential allocation (SA) algorithm, and compare the performance of the SA algorithm with the optimal benchmark for a small test network. We adopt notations and formulations from $[2,27]$.

\subsection{Notation for the Basic MILP}

First, we introduce the optimization objective of the MILP. In the basic RSA problem, the optimization objective, $C$, is the total spectrum usage. In other words, if the lowest frequency assigned is denoted as $0 \mathrm{~Hz}, C$ is the highest frequency assigned in the EONs. 
Second, we introduce the sets used in the MILP. The EON is formulated as an all-pass graph with nodes denoted by $\mathbb{N}$ and unidirectional links denoted by $\mathbb{L}$. $\mathbb{N}$ is the set of all network nodes in the EON. $\mathbb{L}$ is the set of all fiber links in the EON. Each link has its source node $i \in \mathbb{N}$ and destination node $j \in \mathbb{N}$. We denote a certain link by its source and destination, $L_{i, j} \in \mathbb{L}$. The sets of nodes $\mathbb{N}$ and links $\mathbb{L}$ are based on the topology of the EON. $\ell_{i, j}$ is the length of $L_{i, j}$. $\mathbb{D}$ is the set of demands. The set of demands $\mathbb{D}$ is independent of $\mathbb{N}$ and $\mathbb{L}$. In simulations, the demands $\mathbb{D}$ could be generated by a probabilistic model or based on data collected by industry. Each demand needs to be routed from its source node $s \in \mathbb{N}$ to its destination $d \in \mathbb{N}$. The notation for a certain demand from $s$ to $d$ is $D_{s, d} \in \mathbb{D}$. In addition, the required data-rate for $D_{s, d}$ is $R_{s, d}$, where $s \in \mathbb{N}$ and $d \in \mathbb{N}$. $\eta$ is the spectral efficiency, which depends on the modulation format applied to demands.

Third, we introduce the parameters used in the MILP for the basic RSA problem. $\Delta_{s, d}$ is the bandwidth of demand $D_{s, d}$, where $\Delta_{s, d}=\eta \times R_{s, d} . S_{n ; s, d}$ is used for organizing the relationship between nodes in $\mathbb{N}$ and demands $D_{s, d} \in \mathbb{D}$. The parameters $S_{n ; s, d}$ are obtained by the relationship between demands $\mathbb{D}$ and network topology $\mathbb{N}$, L. $S_{n ; s, d}$ is used for flow conversation,

$$
S_{n ; s, d}=\left\{\begin{array}{cl}
1 & \text { if node } n \text { is the source node of demand } S_{n ; s, d} \text { and } n=s \\
-1 & \text { if node } n \text { is the destination node of demand } S_{n ; s, d} \text { and } n=d \\
0 & \text { otherwise }
\end{array}\right.
$$

Lastly, we introduce the decision variables used in the MILP for the basic RSA problem. $F_{s, d}$ denotes the real-valued decision variable that represents the starting frequency allocated to demand $D_{s, d}$. $\mathrm{USE}_{i, j ; s, d}$ denotes the binary decision variable 
that represents link usage corresponding to demand $D_{s, d}$,

$$
\mathrm{USE}_{i, j ; s, d}=\left\{\begin{array}{cl}
1 & \text { if } L_{i, j} \text { is assigned for demand } D_{s, d} \\
0 & \text { if } L_{i, j} \text { is not assigned for demand } D_{s, d}
\end{array} .\right.
$$

$\mathrm{USE}_{i, j ; s, d}$, and $\mathrm{USE}_{i, j ; \hat{s}, \hat{d}}$ are used to represent whether or not the two demands, $D_{s, d}$ and $D_{\hat{s}, \hat{d}}$, are deployed on the same link $L_{i, j} \in \mathbb{L} . \delta_{s, d, \hat{s}, \hat{d}}$ is a binary decision variable that represent the relationship between the spectrum allocated to $D_{s, d}$ and $D_{\hat{s}, \hat{d}}$,

$$
\delta_{s, d, \hat{s}, \hat{d}}=\left\{\begin{array}{cl}
1 & \text { if } F_{s, d} \leq F_{\hat{s}, \hat{d}} \\
0 & \text { if } F_{s, d}>F_{\hat{s}, \hat{d}}
\end{array}\right.
$$

The decision variables $\delta_{s, d, \hat{s}, \hat{d}}, F_{s, d}, \mathrm{USE}_{i, j ; s, d}$ are used to obtain the optimization objective $C$ in an MILP solver.

\subsection{Basic MILP Constraints}

The basic MILP algorithm to solve the RSA problem requires these sets of constraints:

- Total spectrum usage constraint:

$$
C \geq F_{s, d}+\Delta_{s, d}, \quad \forall s, d \in \mathbb{N}
$$

- Flow conservation constraint:

$$
S_{n ; s, d}=\sum_{j=n ; L_{i, j} \in \mathbb{L}} \mathrm{USE}_{i, j ; s, d}-\sum_{i=n ; L_{i, j} \in \mathbb{L}} \mathrm{USE}_{i, j ; s, d}, \quad \forall s, d, n \in \mathbb{N}
$$


- No spectrum overlap constraint:

$$
\begin{aligned}
\delta_{s, d ; \hat{s}, \hat{d}}+\delta_{\hat{s}, \hat{d} ; s, d} & =1, \quad \forall s, d ; \hat{s}, \hat{d} \in \mathbb{N} \\
\left(F_{s, d}-F_{\hat{s}, \hat{d}}+\Delta_{s, d}+\Delta_{g b}\right) & \leq\left(\bar{L}+\Delta_{g b}\right) \times \\
& \left(1-\delta_{s, d, \hat{s}, \hat{d}}+1-\mathrm{USE}_{i, j ; s, d}+1-\mathrm{USE}_{i, j ; \hat{s}, \hat{d}}\right)
\end{aligned}
$$

Equation (4.4) is used to enforce the relationship between the optimization objective $C$ and the highest frequency used in the EON. Equation (4.5) ensures that each demand has only one path from source to destination without bifurcations, loops, or dead-ends during the transmission through intermediate nodes. Equations (4.6) and (4.7) ensure the starting frequencies of each demand are far enough to prevent overlapping. $\bar{L}$ is a large fixed number.

\subsection{QoT Requirements}

We extend the MILP for the basic RSA problem to implement QoT constraints based on the TR and the CLGN models, separately.

\subsubsection{GNTR Model}

The constraints based on the TR model ensure the QoT by limiting the route length when each demand travels from its source to destination node. This thesis extends the basic MILP by adding constraints based on the GNTR model.

$\mathbb{T}$ is the set of transmission reaches corresponding to demands. $\mathrm{T}_{s, d}$ represents the TR for demand $D_{s, d}$. The set $\mathbb{T}$ can be obtained by our proposed GNTR algorithm, given the set $\mathbb{D}$; the algorithm would work for any method used to generate the TR. The GNTR constraint is used to enforce the desired QoT requirements: 
- GNTR constraint

$$
\sum_{\forall L_{i, j} \in \mathbb{L}} \mathrm{USE}_{i, j ; s, d} \times \ell_{i, j} \leq \mathrm{T}_{s, d}, \quad \forall s, d \in \mathbb{N}
$$

Equation (4.8) ensures that for each demand, from its source to destination node, the transmitted length is no longer than the permitted length calculated by the GNTR algorithm. Since $\mathbb{T}$ is pre-calculated by the GNTR model, the multiplication of the decision variable $\mathrm{USE}_{i, j ; s, d}$ and the length of each link $\ell_{i, j}$, can be processed by the MILP engine.

\subsubsection{CLGN Model}

Similarly, we extend the MILP for the basic RSA problem to implement the QoT constraints based on the CLGN model.

$\operatorname{SINR}_{s, d}^{t h}$ denotes the required SINR for a given demand $D_{s, d}$ and a specified QoT, corresponding to $\eta$. $\eta$ represents the spectral efficiency of the modulation chosen. The $\mathrm{SINR}_{s, d}^{t h}$ and pre-FEC BER thresholds are consistent with the required SINR and pre-FEC BER threshold used in the GNTR algorithm.

The following constraints are needed to incorporate the CLGN into the basic MILP:

- ASE PSD for demand $D_{s, d}$ :

$$
G_{A S E ; s, d}=\sum_{\left(L_{i, j} \in \mathbb{L}\right) \cap\left(\mathrm{USE}_{i, j ; s, d}=1\right)} \frac{\ell_{i, j}}{L} \times G_{A S E ; s, d}^{s p a n}, \quad \forall s, d \in \mathbb{N}
$$


- NLI PSD for demand $D_{s, d}$ :

$$
G_{N L I ; s, d}=\sum_{\left(L_{i, j} \in \mathbb{L}\right) \cap\left(\mathrm{USE}_{i, j ; s, d}=1\right)} \frac{\ell_{i, j}}{L} \times \mathbf{C L}\left(G_{N L I ; s, d}^{s p a n}\right), \quad \forall s, d \in \mathbb{N}
$$

- QoT constraint based on the CLGN model:

$$
G_{A S E ; s, d}+G_{N L I ; s, d} \leq \frac{G_{s, d}}{\operatorname{SINR}_{s, d}^{t h}}, \quad \forall s, d \in \mathbb{N}
$$

where the $G_{s, d}$ is the input PSD for demand $D_{s, d}$. Equation (4.9) obtains the ASE PSD by summing up all links on the route selected for demand $D_{s, d} \in \mathbb{D}$. $G_{A S E ; s, d}$ is the ASE PSD accumulated along the route. The ASE noise only depends on

the length that the transmitted signal travels. In (4.10), $\mathbf{C L}\left(G_{N L I ; s, d}^{s p a n}\right)$ is the NLI PSD per span, calculated by the CLGN model. Moreover, this equation sums up the propagation distance of $D_{s, d}$ in order to obtain the NLI PSD accumulated along the route, denoted as $G_{N L I ; s, d}$. Equation (4.11) enforces that each deployed demand should satisfy the desired QoT.

\subsection{MILP with Regeneration Nodes}

In Section 4.3 of this thesis, we extended the MILP for the basic RSA model to implement the QoT requirements. Because of the accumulated noise along the route, a demand might not be able to route from its source to destination and still satisfy the QoT constraints. Therefore, when we apply these two models in large scale network topologies, such as the NSF-24, the MILP may not yield a viable solution. Hence, we need to consider using regeneration nodes to negate the accumulated noise effects and attain the desired QoT.

To model the regeneration nodes and their OEO function in the MILP formula- 
tions, this thesis assumes the following facts for the GNTR model and the CLGN model:

1. In the GNTR model, the transmission reach can be extended after the signal is regenerated at the regeneration nodes. In other words, the propagation distance accumulated along the route becomes zero when the demand passes the regeneration node and uses a regeneration circuit.

2. In the CLGN model, the total noise caused by ASE and NLI accumulated along the route becomes zero when the light-path passes the regeneration node and uses a regeneration circuits. The accumulated noise along the route is canceled by the regeneration nodes with the input PSD unchanged.

Based on the two assumptions above, we can extend the MILP described in Section 4.3 to incorporate regeneration nodes. We use existing formulations from [2] for modeling signal regeneration. After adding constraints at signal regeneration nodes, these two MILPs, the GNTR-model-based MILP and the CLGN-model-based MILP, are able to function for practical continental-scale networks. With regeneration nodes, the optimization objective becomes a multi-objective function with a weighting factor, either $C+\varepsilon T$ or $T+\varepsilon C$, where $\varepsilon$ is a small number, $C$ is still the spectrum used, and $T$ is the number of regeneration nodes. Note that basic assumptions are listed as follows: no wavelength conversion, no modulation conversion, and all demands use the same modulation format.

\subsubsection{GNTR Model with Regeneration Nodes}

$\mathbf{Y}_{n ; s, d}^{\mathrm{TR}}$ denotes a real-valued decision variable that represents the physical propagation distance. $\mathbf{Y}_{n ; s, d}^{\mathrm{TR}}=0$ when demand $D_{s, d}$ does not route through node $n$. Otherwise, $\mathbf{Y}_{n ; s, d}^{\mathrm{TR}}$ is the physical propagation distance of demand $D_{s, d}$ from the beginning 
node of the transparent segment to node $n \in \mathbb{N} . \mathbf{z}_{i, j ; s, d}^{\mathrm{TR}}$ is a real-valued decision variable that represents the relationship between the physical traveling distance $\mathbf{Y}_{n ; s, d}^{\mathrm{TR}}$ and the usage of the link $L_{i, j}, \mathrm{USE}_{i, j ; s, d} \cdot \mathbf{z}_{i, j ; s, d}^{\mathrm{TR}}=0$ if demand $D_{s, d}$ is not assigned on link $L_{i, j}$, i.e., if $\mathrm{USE}_{i, j ; s, d}=0$. Otherwise, $\mathbf{z}_{i, j ; s, d}^{\mathrm{TR}}$ is the accumulated propagation distance from the beginning node of the transparent segment on the light-path to node $i$, i.e., $\mathbf{z}_{i, j ; s, d}^{\mathrm{TR}}=\mathbf{Y}_{i ; s, d}^{\mathrm{TR}}$, where $i \in \mathbb{N}$ is on the route of demand $D_{s, d} . I_{n ; s, d}$ denotes a Boolean decision variable representing the allocation of a regeneration circuit on node $n \in \mathbb{N}$, corresponding to demand $D_{s, d}$. The regeneration nodes contain a limited number of regeneration circuits and the maximum number of regeneration circuits used per regeneration node is $I^{\max }$. $I_{n ; s, d}=1$ if a regeneration circuit for a demands $D_{s, d}$ is allocated on node $n$. Otherwise, $I_{n ; s, d}=0$. $I_{n}$, a Boolean decision variable, represents whether node $n$ is a regeneration node or not. $I_{n}$ is related to $I_{n ; s, d}$ (for $I_{n ; s, d}=1$, node $n$ has to be a regeneration node, i.e., $I_{n}=1$, and its regeneration circuit has to be used by demand $D_{s, d}$ ). If $D_{s, d}$ needs a regeneration circuit, node $n$ has to be a regeneration node. $\mathbf{k}_{i, j ; s, d}^{\mathrm{TR}}$ denotes a real-valued decision variable that manages the relationship between the regeneration node $I_{n}$ and the accumulated length $\mathbf{z}_{i, j ; s, d}^{\mathrm{TR}}$. If node $n$ is a regeneration node and the regeneration circuit is needed, $I_{n ; s, d}=1$, then $\mathbf{k}_{i, j ; s, d}^{\mathrm{TR}}=0$. Otherwise, $\mathbf{k}_{i, j ; s, d}^{\mathrm{TR}}=\mathbf{z}_{i, j ; s, d}^{\mathrm{TR}}$, which means that demand $D_{s, d}$ is not regenerated at node $n$ and the accumulated length is not affected.

The resulting constraints that must be added to the MILP to implement regeneration nodes are:

- Link usage and accumulated traveling distance constraint:

$$
\mathbf{z}_{i, j ; s, d}^{\mathrm{TR}}=\mathbf{Y}_{n ; s, d}^{\mathrm{TR}} \times \mathrm{USE}_{i, j ; s, d} \quad, \forall i, j, s, d \in \mathbb{N}
$$


- Transmission reach constraint (QoT constraint of the GNTR model:

$$
\mathbf{Y}_{n ; s, d}^{\mathrm{TR}} \leq \mathrm{T}_{s, d}, \quad \forall s, d, n \in \mathbb{N}
$$

- Accumulated traveling distance constraint:

$$
\mathbf{Y}_{n ; s, d}^{\mathrm{TR}}=\sum_{L_{i, j} \in \mathbb{L} ; j=n} \mathbf{k}_{i, j ; s, d}^{\mathrm{TR}}+\mathrm{USE}_{i, j ; s, d} \times \ell_{i, j}, \quad \forall s, d, n \in \mathbb{N}
$$

- Regeneration circuits and accumulate length constraint:

$$
\mathbf{k}_{n ; s, d}^{\mathrm{TR}}=\left(1-I_{n ; s, d}\right) \times \mathbf{z}_{i, j ; s, d}^{\mathrm{TR}}, \quad \forall s, d, n \in \mathbb{N}
$$

- Regeneration circuits and regeneration nodes constraint:

$$
I_{n} \times I^{\max } \geq \sum_{\forall D_{s, d} \in \mathbb{D}} I_{n ; s, d}, \quad \forall n \in \mathbb{N}
$$

Equation (4.12) builds the relationship between $\mathbf{Y}_{n ; s, d}^{\mathrm{TR}}$ and $\mathbf{z}_{i, j ; s, d}^{\mathrm{TR}}[44]$. Equation (4.13) is used to replace constraint (4.8) when regeneration nodes are employed. It ensures that a demand is transmitted with the desired QoT. Equation (4.14) is used to obtain $\mathbf{Y}_{n ; s, d}^{\mathrm{TR}}$ by a recursive accumulation of the propagation distance along the route of the demand. Equation (4.15) is used to ensure the relationship between regeneration circuits and the accumulated length. Equation (4.16) ensures the number of regeneration circuits on one regeneration node is bounded by the maximum allowed, $I^{\max }$. In addition, we assume that if there is a regeneration circuit on node $n$, then node $n$ must be a regeneration node. 


\subsubsection{CLGN Model with Regeneration Nodes}

$\mathbf{Y}_{n ; s, d}^{C L}$ denotes a real-number decision variable representing the accumulated noise, both ASE and NLI, along the route corresponding to demand $D_{s, d}$ from the beginning node of the transparent segment on the light-path to node $n \in \mathbb{N}$. $\mathbf{Y}_{n ; s, d}^{C L}=0$ if the demand does not route through node $n$. Otherwise, $\mathbf{Y}_{n ; s, d}^{C L}$ is the sum of the ASE and NLI noise along the route from its last regeneration node to node $n . \mathbf{z}_{i, j ; s, d}^{C L}$, also a real valued variable, represents the relationship between the accumulated noise $\mathbf{Y}_{n ; s, d}^{C L}$ and the link usage $\mathrm{USE}_{i, j ; s, d} \cdot \mathbf{z}_{i, j ; s, d}^{C L}=0$ if demand $D_{s, d}$ is not assigned to link $L_{i, j}$, i.e., $\mathrm{USE}_{i, j ; s, d}=0$. Otherwise, $\mathbf{z}_{i, j ; s, d}^{C L}$ is the accumulated noise from the beginning node of the transparent segment on the light-path to node $i \in \mathbb{N}$, and $i$ is on the route of demand $D_{s, d}$, i.e., $\mathbf{z}_{i, j ; s, d}^{C L}=\mathbf{Y}_{i ; s, d}^{C L} . \quad \mathbf{k}_{i, j ; s, d}^{C L}$ is a real-valued decision variable that manages the relationship between regeneration node $n$ and the accumulated total noise $\mathbf{z}_{i, j ; s, d}^{C L}$. If node $n$ is a regeneration node and the regeneration circuit is needed, $I_{n ; s, d}=1$, then $\mathbf{k}_{i, j ; s, d}^{C L}=0$. Otherwise, $\mathbf{k}_{i, j ; s, d}^{C L}=\mathbf{z}_{i, j ; s, d}^{C L}$, which means demand $D_{s, d}$ is not regenerated at node $n$ and the accumulated length is not be affected.

The resulting constraints that must be added to the MILP to implement regeneration nodes are:

- Link usage and accumulated noise constraint:

$$
\mathbf{z}_{i, j ; s, d}^{C L}=\mathbf{Y}_{n ; s, d}^{C L} \times \mathrm{USE}_{i, j ; s, d}, \quad \forall s, d, i, j \in \mathbb{N}
$$

- QoT constraint of the CLGN model:

$$
\mathbf{Y}_{n ; s, d}^{C L} \leq \frac{G_{s, d}}{\operatorname{SINR}_{s, d}^{t h}}, \quad \forall s, d, n \in \mathbb{N}
$$


- Accumulated noise constraint:

$$
\mathbf{Y}_{n ; s, d}^{C L}=\sum_{L_{i, j} \in \mathbb{L} ; j=n} \mathbf{k}_{i, j ; s, d}^{C L}+\mathrm{USE}_{i, j ; s, d} \times \frac{\ell_{i, j}}{L}\left(G_{A S E ; s, d}+G_{N L I ; s, d}\right), \quad \forall s, d, n \in \mathbb{N}
$$

- Regeneration circuits and accumulated noise constraint:

$$
\mathbf{k}_{n ; s, d}^{C L}=\left(1-I_{n ; s, d}\right) \times \mathbf{z}_{i, j ; s, d}^{C L}, \quad \forall s, d, n \in \mathbb{N}
$$

- Regeneration circuits and regeneration nodes constraint:

$$
I_{n} \times I^{\max } \geq \sum_{\forall D_{s, d} \in \mathbb{D}} I_{n ; s, d}, \quad \forall n \in \mathbb{N}
$$

Equation (4.18) is used to replace (4.11) when regeneration nodes are employed. It ensures that each demand transmitted satisfies the desired QoT. Equation (4.19) is used to obtain $\mathbf{Y}_{n ; s, d}^{C L}$ by a recursive accumulation of the total noise along the route. $I^{\max }$ in (4.21) is again the maximum number of regeneration circuits used on each regeneration node.

\subsection{Heuristic Algorithm: Sequential Allocation}

\subsubsection{Motivation of the Sequential Allocation}

With the development of modern communication techniques leading to expansion of the number of demands (such as more users involved in communications) and the dimensions of topologies (such as a larger scale inter-continental backbone networks) in EON, the complexity of the RSA problem goes beyond the capacity of current 
solvers. Even though the CLGN model provides less complexity than the standard GN model, the RSA problem cannot be properly solved within a reasonable time, using the standard MILP algorithm. For example, for large network topologies, such as the NSF-24 network with 24 nodes and 86 links, or the DT-14 network with 14 nodes and 46 links, current computational resources are not able to successfully solve the RSA problem. Therefore, heuristic algorithms are used to provide acceptable sub-optimal solutions to these complex RSA problems.

The heuristic algorithm we propose is called the sequential allocation (SA) algorithm. The SA algorithm is a recursive and iterative method to solve the RSA problems and saves on computational resource consumption. It optimizes the routing of the demands and the assignment of spectral resources simultaneously and recursively. The merit of the recursiveness is to shrink the number of variables and to provide a near-optimal starting point at each iteration.

MILP problems are much more complex than basic linear programming problems because, in MILP problems, there are integers that have to be solved by the method of exhaustion. Therefore, for optimal MILP methods, during the solving process an unacceptably long time is spent solving for the integers values in intermediate results. These scenarios are likely to be considered as impasses of the optimization processes. The SA algorithm can mostly avoid these impasses, where the optimal MILP methods are usually overwhelmed. The SA algorithm reduces the number of integer variables, thus leading to savings in computational resources by using iterations. In addition, the SA algorithm applies randomness in order to enhance the performance of each iteration. The randomness allows the algorithm to explore many non-contiguous areas of the variable space, resulting in a closer approach to the optimal solution. 


\subsubsection{SA Process}

The process of the SA algorithm is shown in Algorithms 1-5.

In Algorithm 1, when the number of demands optimized is less than the number of demands needing to be optimized (i.e., $\mathbb{D}^{*} \neq \mathbb{D}$ ), we iteratively expand the size of the optimization problem (i.e., $\mathbb{D}^{*} \cup \mathbb{D}_{\text {new }}=$ AddNewDemands $\left(\mathbb{D}^{*}, \mathbb{D}, m\right)$ ) and optimize it, until the expanded size equals the desired size (i.e., $\mathbb{D}^{*}=\mathbb{D}$ ). In addition, between two expanding process (i.e., the number of demands $\left|\mathbb{D}^{*}\right|$ remains unchanged), we reoptimize the problem (with the number of demands unchanged) for $N$ iterations by applying randomness to each iteration.

In Algorithm 2, when the number of optimized demands is less than the number of demands needing to be optimized $\left(\mathbb{D}^{*} \neq \mathbb{D}\right)$, we add $m$ more demands into the optimization problem. The demands to be used in the optimization process (i.e., Algorithm 4) become $\mathbb{D}^{*} \cup \mathbb{D}_{\text {new }}$, where $\left|\mathbb{D}_{\text {new }}\right|=m$.

In Algorithm 3, we randomly select a set of demands, $\mathbb{D}_{\text {out }}$ from the set of optimized demands, $\mathbb{D}^{*}$. The size of the selected demand set is half of that of the optimized demands (i.e., $\left|\mathbb{D}_{\text {out }}\right|=\left\lfloor\left|\mathbb{D}^{*}\right| / 2\right\rfloor$ ). We then apply the remaining selected demands into Algorithm 5.

In Algorithm $4, \mathbb{D}^{*} \cup \mathbb{D}_{\text {new }}$ represent the set of demands that needs to be optimized. $\delta\left(\mathbb{D}^{*}\right)$ represent the optimized spectrum information obtained from the last iteration when the set of demands is $\mathbb{D}^{*}$. USE $\left(\mathbb{D}^{*}\right)$ represents the optimized routing information obtained from the previous iteration when the set of demands is $\mathbb{D}^{*}$. This MILP process optimizes the expanded demands set $\left(\mathbb{D}^{*} \cup \mathbb{D}_{\text {new }}\right)$ based on the optimized spectrum and routing information from the optimized demands set $\mathbb{D}^{*}$.

In Algorithm $5, \mathbb{D}_{\text {out }}$ represent the set of demands randomly selected from the optimized set of demands $\mathbb{D}^{*}$. In this algorithm, we delete the routing $\operatorname{USE}\left(\mathbb{D}_{\text {out }}\right)$ 





and spectrum assignment information $\delta\left(\mathbb{D}_{\text {out }}\right)$ from the last iteration with demands $\mathbb{D}^{*}$. Based on the remaining spectrum and routing information, i.e., $\delta\left(\mathbb{D}^{*} \backslash \mathbb{D}_{\text {out }}\right)$ and $\operatorname{USE}\left(\mathbb{D}^{*} \backslash \mathbb{D}_{\text {out }}\right)$, the demands $\mathbb{D}^{*}$ are reoptimized again. In this reoptimization process, demands are optimized based on partially optimized information from the previous iteration.
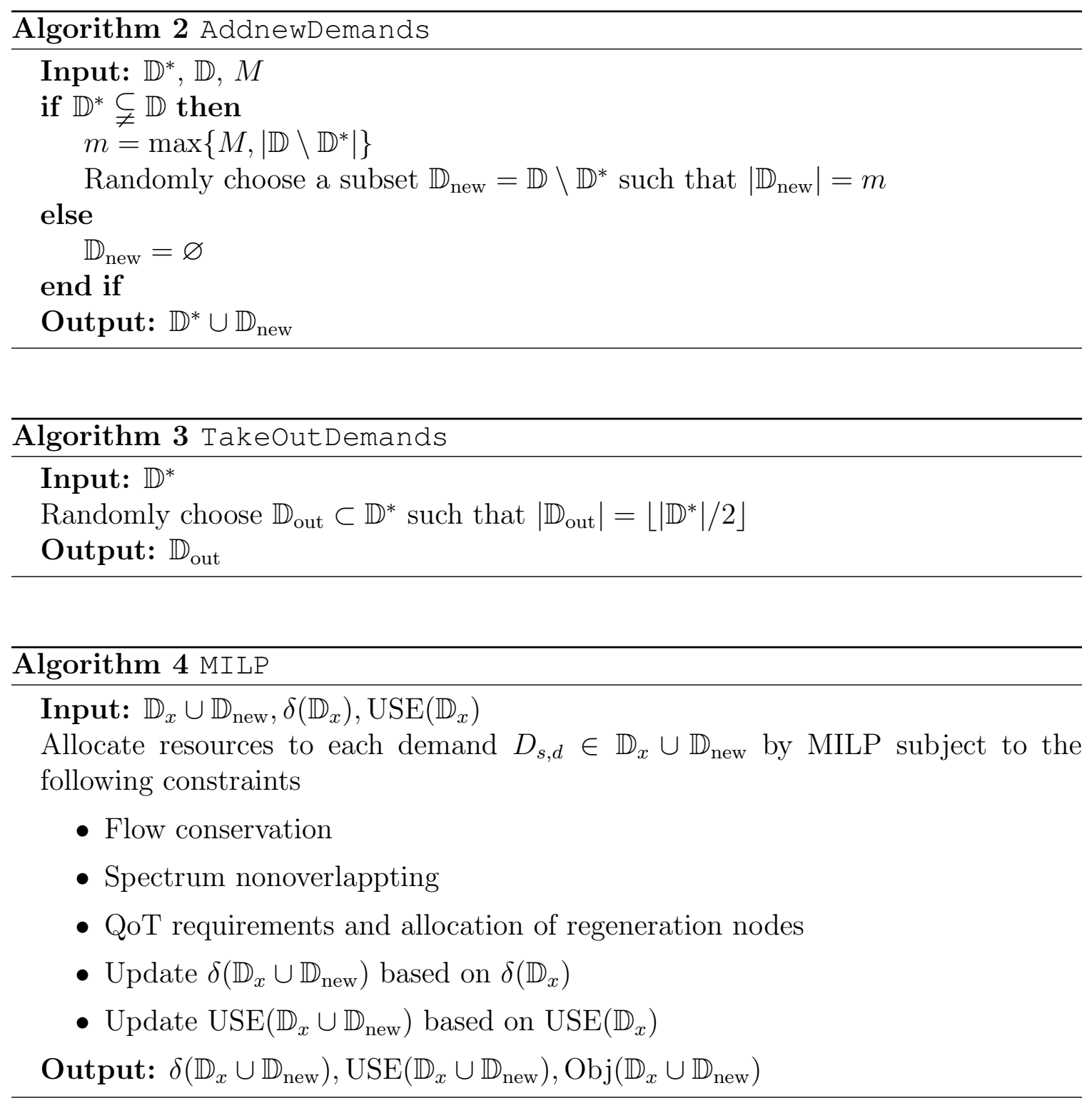




\subsection{Comparison with the Benchmark}

In order to measure the performance of the proposed SA algorithm, this thesis applies the optimal MILP as a benchmark for comparison. Simulations for both the optimal MILP and the SA algorithm are based on a six node network, shown in Figure 4.1. The network parameters are listed in Table 3.1. We apply the GNTR model and the CLGN model separately for both the optimal MILP and the SA algorithm. The optimization objective in this scenario is to minimize the total spectrum usage, $C$. No regeneration is assumed. We generate static traffic demands between node pairs, wherein each pair consists of a source and a destination node. For every pair, each node is uniformly selected. The bandwidth of these demands is uniformly distributed in the range from 30 to $100 \mathrm{GHz}$. Identical demands are deployed for both the SA and the optimal MILP algorithms. Binary phase shift keying (BPSK) modulation is used for all demands [2]. All simulation results lie within 90\% confidence intervals. Note that the simulation settings are similar to common assumptions used in literature $[2-$ 4].

In Figure 4.2, after 30 simulation trials, we find that when the number of demands 




Figure 4.1: Six node test network [4]. The number on each link corresponds to the number of spans.

ranges from 5 to 20, both the optimal MILP and the SA algorithm provide identical results (the spectrum assignment is identical, but since the network topology is symmetrical the routing can be different). We thus conclude that the solutions obtained by the SA algorithm can achieve the global optimum for small dimension networks. 


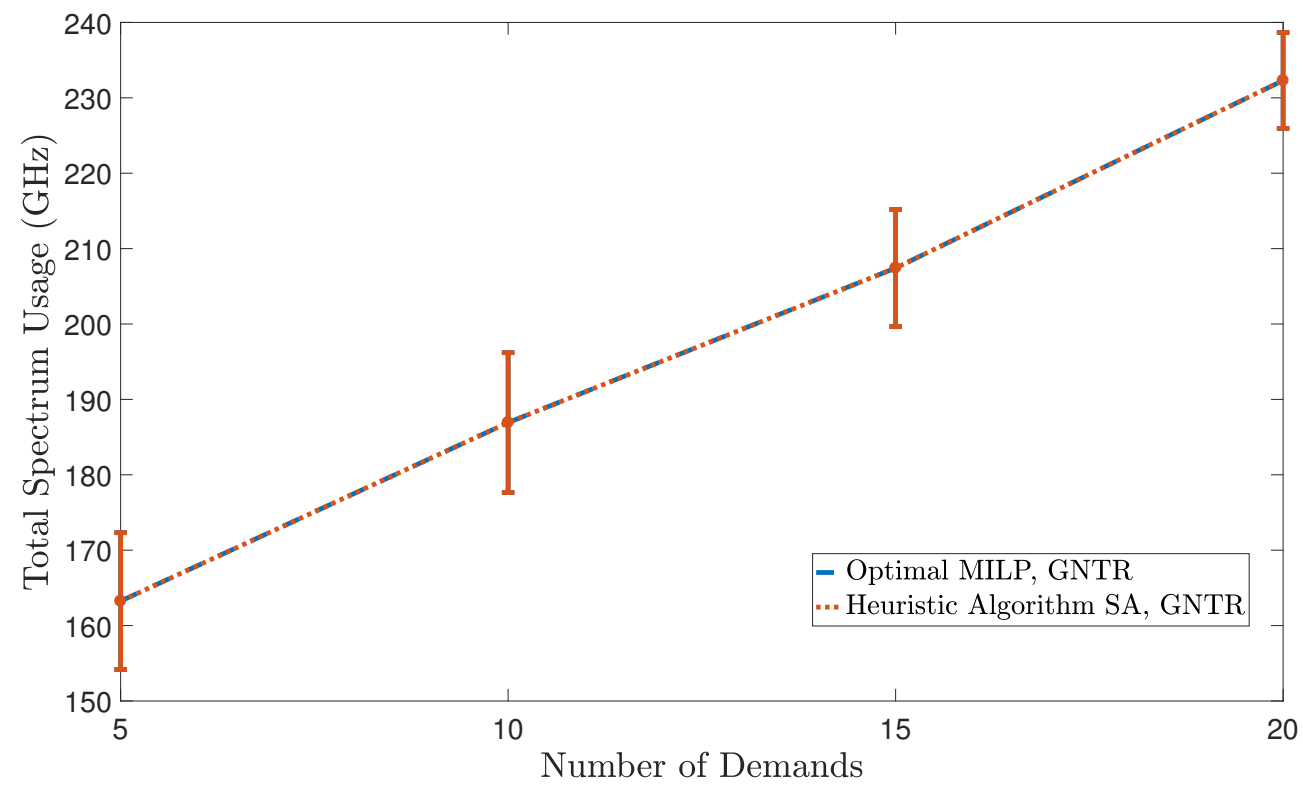

(a)

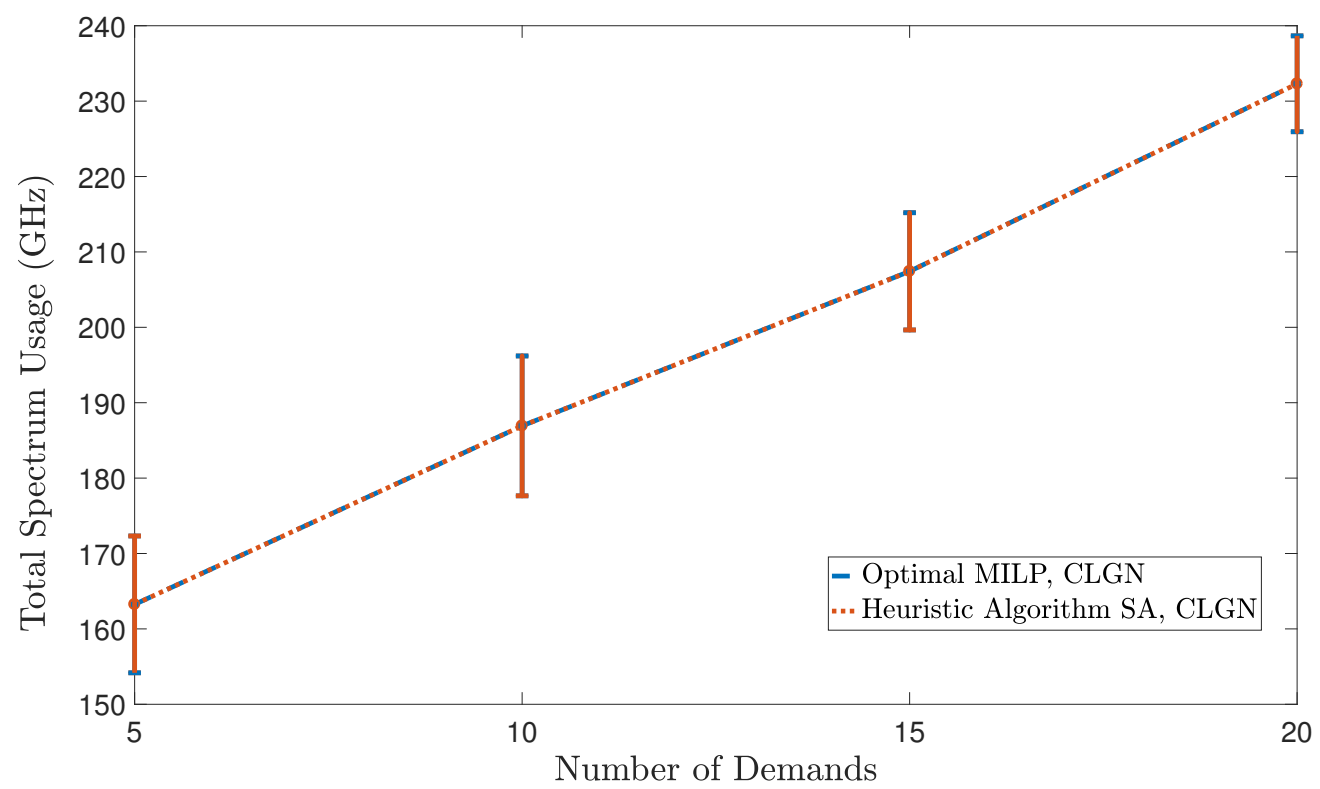

(b)

Figure 4.2: Total spectrum usage versus number of demands for optimal MILP and proposed heuristic algorithm SA in 6-node test network, with BPSK modulation. (a) GNTR model. (b) CLGN model. 


\section{Chapter 5}

\section{Numerical Results}

In this chapter, through simulation of different scenarios of the RSA problem, we demonstrate the merits of the CLGN model compared with the GNTR model for realistic networks.. We also show the advantages of the proposed SA algorithm compared with existing heuristic algorithms.

\subsection{Simulation Settings}

We ran our simulations on the Rivanna research computing cluster provided by the University of Virginia [45]. The MILP engine used for simulation is the Gurobi Optimization [46]. The fiber parameters for our simulation are based on Table 3.1. The network topologies we tested are the NSF-24 network with 24 nodes and 86 unidirectional links, shown in Figure 5.1, and the DT-14 network with 14 nodes and 46 unidirectional links, shown in Figure 5.2. These two network topologies are used for testing algorithms with different scalability (the DT-14 network is used for less scalable algorithms, and the NSF-24 network is used for more scalable algorithms). All plots show the mean values of the quantity measured and $90 \%$ confidence intervals. We generate static traffic demands between node pairs, wherein each pair consists of 




Figure 5.1: DT-14 network [4]. The number on each link corresponds to the number of spans.

a source and a destination node. For every pair, each node is randomly selected. The bandwidth of these demands is uniformly distributed from 30 to $100 \mathrm{GHz}$. All demands use the same modulation format (either BPSK or QPSK). No modulation conversion or wavelength conversion is considered [2]. Again, the simulation settings are similar to common assumptions used in literature [2-4].

\subsection{Results for DT-14 Network}

In this scenario, we call the combination of our proposed CLGN model and SA heuristic algorithm the proposed system. This system models the PLIs using the CLGN model and solves the RSA problem by using the SA algorithm. We compare our proposed system with another published system presented in [3] that models the RSA problem by a finer linearized GN model and solves the problem by MILP. We consider the system published in [3] as a benchmark, and compare the proposed 


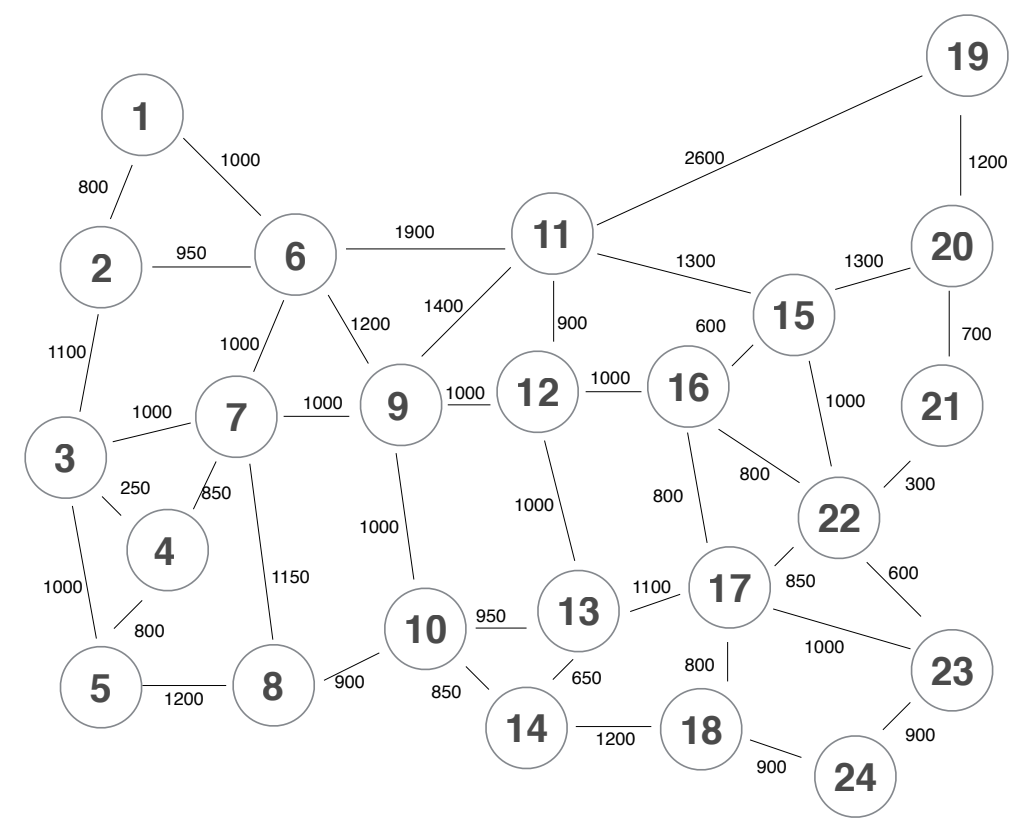

Figure 5.2: NSF-24 network [2]. The number on each link represents the physical length of the link in $\mathrm{km}$.

system with it. We apply the same topology, the DT-14 network, and the same demands for both systems. The DT-14 network is chosen over the NSF-24 network in this section due to the computational limitations of the benchmark system, which is not able to find a solution for large network topologies or a large number of demands. Since the network is relatively small in scale, we do not consider any regeneration $(T=0)$. The objective here for both systems is to minimize the total spectrum usage, $C$.

In Figure 5.3 (a), the total spectrum usage required by our proposed system is slightly higher than that of the published system. When the number of demands is less than or equal to 15 , the proposed system and the benchmark system have similar spectrum usage. When the number of demands is between 15 to 30, the gap in the required spectrum between the proposed system and the published system is less than $10 \%$. When the number of demands exceeds 30 , the published system fails to give 


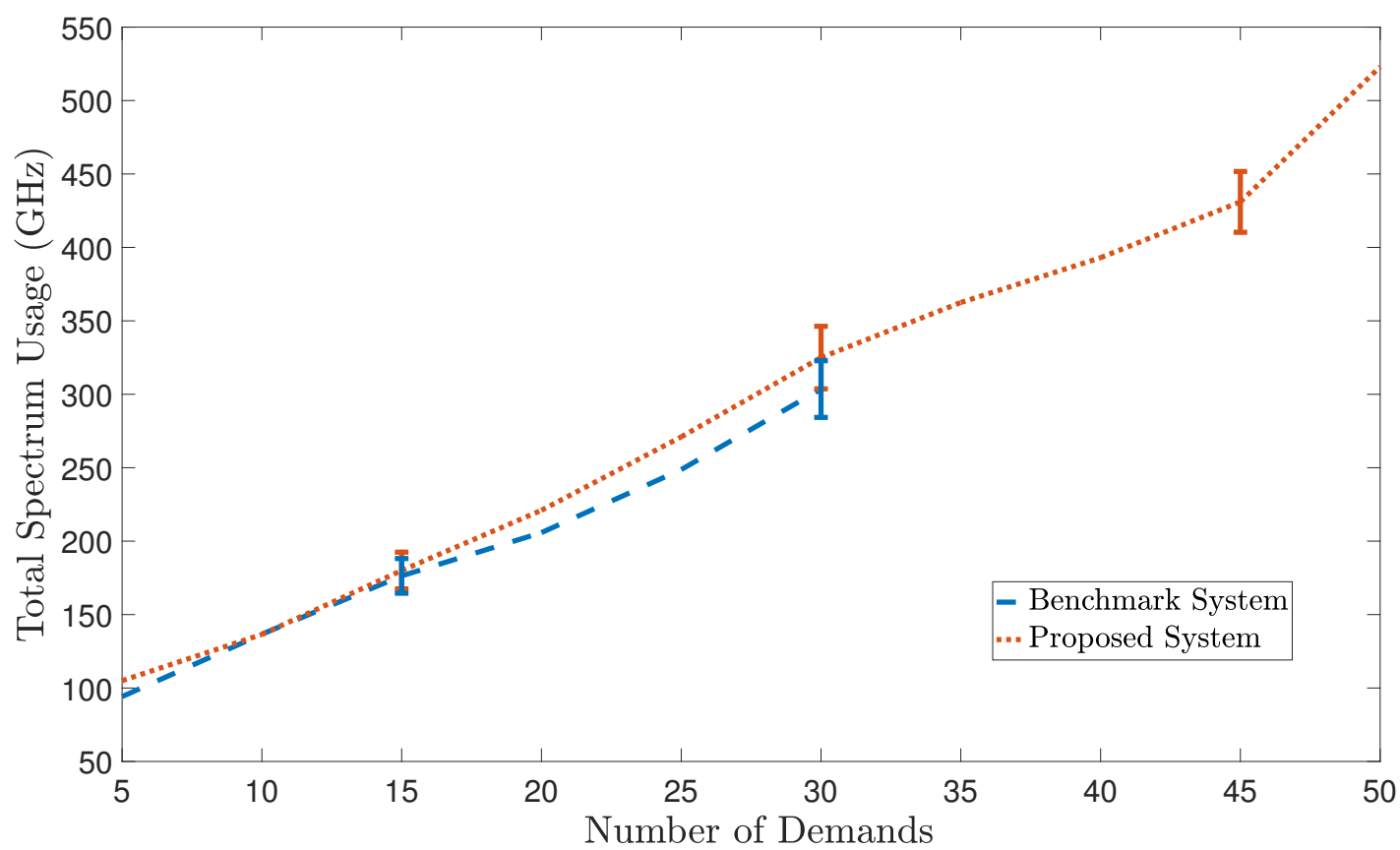

(a)



(b)

Figure 5.3: (a) Total spectrum usage versus number of demands for proposed system and benchmark system in DT-14 network, with BPSK modulation. (b) Elapsed time versus number of demands for proposed system and benchmark system in DT-14 network. 
any result within a reasonable time (i.e., 36 hours). However, the proposed system is able to solve the RSA problem. Although the benchmark system provides better results for up to 30 demands, the proposed system is uniquely able to provide results for up to 50 demands.

In Figure $5.3(\mathrm{~b})$, the total elapsed time for both systems is shown to increase exponentially as a function of the number of demands. However, the computational efficiency of the proposed system is much greater than the benchmark system, due to the SA algorithm appropriately breaking down the RSA problem into smaller problems. These results make the proposed system more applicable to practical scenarios.

We thus conclude that there is a trade-off between the optimality of the spectrum usage and the efficiency in time consumption. Compared to the benchmark, the proposed system sacrifices less than $10 \%$ of the spectrum used to gain the benefits of less complexity in solving the RSA problem, less computation time, and the capability to process the RSA problem deployed with a large number of demands.

\subsection{Results for NSF-24 Network}

To test our algorithms in a continental-sized network, we simulate the NSF-24 topology. This network has been used extensively in literature to evaluate network planning algorithms $[2,47]$.

\subsubsection{RSA with Multi-Optimization Objectives}

Spectrum and regeneration nodes are valuable resources when considering the capital expenditures of deploying long-haul optical networks. In different scenarios, there are different priorities for these resources. We simulate the following representative scenarios to show the performance of our proposed CLGN model and the SA 
algorithm. We tested two objective functions:

1. RSA with objective: $\min C+\varepsilon T$

The objective in this scenario is to minimize the total spectrum usage $C$ plus the total number of regeneration nodes $T$ (i.e. $C+\varepsilon T$ ), where $\varepsilon$ is a small number. Because $C$ is much bigger than $\varepsilon T$, the prime objective here is to optimize the total spectrum usage, $C$. We minimize the total spectrum usage while trying to reduce the number of regeneration nodes. We apply both the GNTR model and the CLGN model, as described in Section 3.6, and solve the RSA problem by the SA algorithm in the NSF-24 network.

2. RSA with objective: $\min T+\varepsilon C$

The objective in this scenario is to minimize the number of total regeneration nodes, $T$, plus the total spectrum usage, $C$. This time the spectrum usage is weighted by a very small number $\varepsilon$. Hence, the prime objective for this optimization scenario is to minimize the number of regeneration nodes while controlling the total spectrum usage with lower priority. We apply the same simulation settings used as in the prior case for simulations in this scenario.

As shown in Figures 5.4 (a) and (b), both objectives above monotonically increase as the number of demands increases. For the $T+\varepsilon C$ case, the CLGN model yields a 37\% savings in optimization objective compared with the GNTR model. However, surprisingly, for the $C+\varepsilon T$ case, the curves of the CLGN model and the GNTR model show little difference; because, as shown below, the spectrum usage for both models is the same, we can attribute this result to the number of regeneration nodes needed, $T$.

In Figures 5.5 (a) and (b), for the $C+\varepsilon T$ case, for both BPSK and QPSK modulations, the total spectrum usage of the GNTR and CLGN models is similar, 


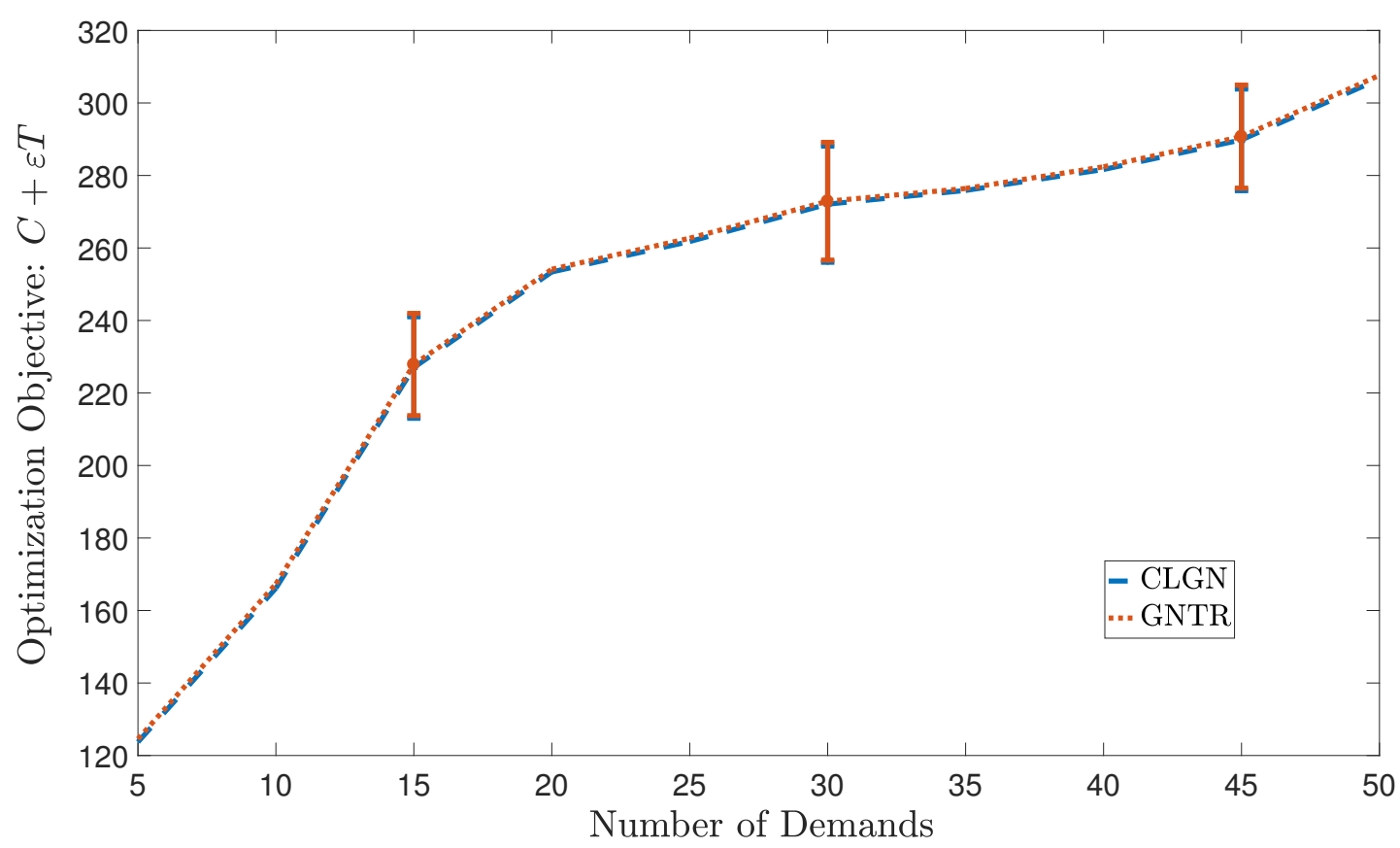

(a)



(b)

Figure 5.4: (a) Optimization objective $C+\varepsilon T$ versus number of demands in NSF-24 network, with QPSK modulation. (b) Optimization objective $T+\varepsilon C$ versus number of demands in NSF-24 network, with QPSK modulation. 
which happens because the total spectrum used is the primary optimization objective. Both the TR and the CLGN models sacrifice other resources, such as the number of regeneration nodes, to ensure the optimality of the spectrum usage. In other words, in this scenario, the difference of the performance caused by the different estimates of the PLIs is compensated by the allocation of regeneration nodes.

In Figure 5.5 (a), for the $T+\varepsilon C$ scenario with BPSK, the loose QoT requirements (the SINR threshold of BPSK is much smaller than the actual SINR) result in similar spectrum used of both the GNTR model and the CLGN model. Comparing the different optimization objectives, $C+\varepsilon T$ and $T+\varepsilon C$, using BPSK, the spectrum usage is similar because the minimum number of regeneration nodes is 0 and the QoT requirements are loose.

In Figure 5.5 (b), for the $T+\varepsilon C$ scenario with QPSK, the total spectrum usage of the GNTR model with less than 20 demands is higher than that of the CLGN model. When the number of demands increases, the value of the total spectrum usage of both the GNTR model and the CLGN model closely align. The reason for this is that the main objective in this scenario is to minimize the number of regeneration nodes. Therefore, when there are fewer than 20 demands, the CLGN model has the potential to save more spectrum, because the CLGN model is state-dependent thus has a more accurate approximation of the noise level. However, the GNTR model is a worst case approximation and overestimates the PLIs when realistic situations are far from the worst case (the actual length a signal can propagate while satisfying the SINR threshold is much longer than the length obtained using the GNTR model). When there are more than 20 demands, both the GNTR and the CLGN models result in the same routing solution in order to save on the number of regeneration nodes, resulting in the curve of the GNTR model closely following the curve of the CLGN model. Comparing the different optimization objectives, $C+\varepsilon T$ and $T+\varepsilon C$, using 




(a)



(b)

Figure 5.5: Total spectrum usage versus number of demands with different optimization objectives $C+\varepsilon T$ and $T+\varepsilon C$ in NSF-24 network. (a) BPSK modulation. (b) QPSK modulation. 
QPSK, the spectrum usage of $T+\varepsilon C$ is higher than that of $C+\varepsilon T$.

Comparing Figures 5.5 (a) and (b), the BPSK and QPSK curves are similar to each other for $C+\varepsilon T$, because the allocation of regeneration nodes compensates the difference between SINR thresholds. Additionally, in both modulation formats, the bandwidth of demands is similarly distributed. Thus, the CLGN model is better in saving total spectrum usage compared with the GNTR model for both modulation formats.

In Figure $5.6(\mathrm{a})$, for the $C+\varepsilon T$ case, the regeneration nodes usage of GNTR is higher than that of CLGN for any number of demands. For the $T+\varepsilon C$ case, the primary optimization objective is to minimize the number of regeneration nodes $T$. Although the curve of the GNTR model is still higher than for the CLGN model, the magnitude of separation is less compared with the $C+\varepsilon T$ case, because the objective is to minimize the number of regeneration nodes. It can also be observed, for the case $T+\varepsilon C$, when demands are fewer than 25, the magnitude of separation between the GNTR and CLGN model curves is greatest. This results from the fact that the GNTR model has more PLIs approximation error when the actual noise level is far from the worst case. Thus, we can conclude that the CLGN model is able to reduce the number of regeneration nodes used compared with the GNTR model.

In Figure 5.6 (b), the number of regeneration circuits is not the optimization objective of either the $C+\varepsilon T$ case or the $T+\varepsilon C$ case. However, regeneration circuits are also an expensive and limited resource in EONs, in addition to regeneration nodes. The GNTR model requires more regeneration circuits compared with the CLGN model in both cases. Additionally, the magnitude of separation between the CLGN and GNTR model curves in the $C+\varepsilon T$ case is larger than that of the $T+\varepsilon C$ case. The reason is that when the number of demands increases, in both the $C+\varepsilon T$ case and the $T+\varepsilon C$ case, the GNTR model results in higher PLIs estimation error, 


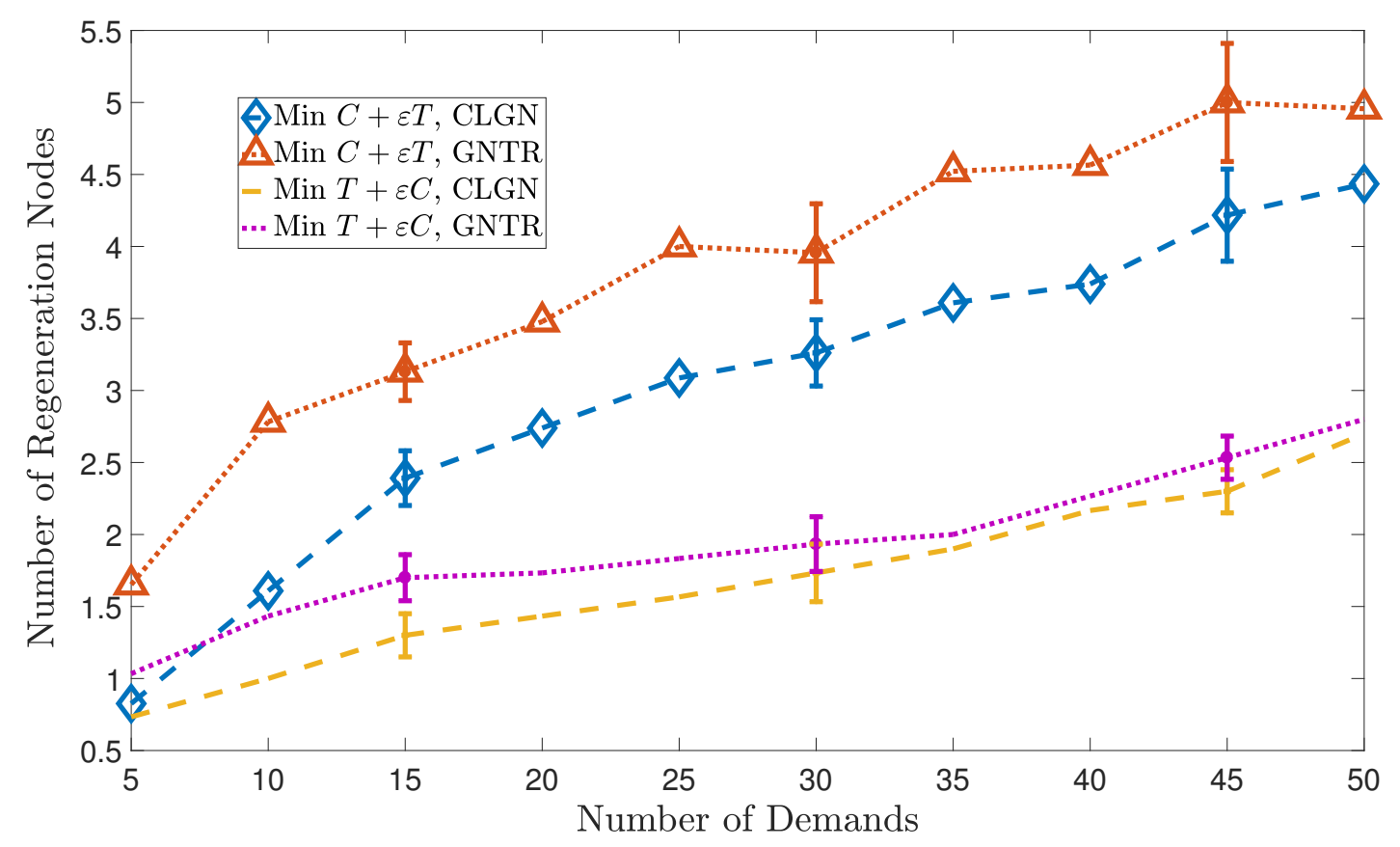

(a)



(b)

Figure 5.6: (a) Number of regeneration nodes versus number of demands with different optimization objectives $C+\varepsilon T$ and $T+\varepsilon C$ in NSF-24 network, with QPSK modulation. (b) Number of regeneration circuits versus number of demands with different optimization objectives $C+\varepsilon T$ and $T+\varepsilon C$ in NSF-24 network, with QPSK modulation. 
leading to extra expenditure of regeneration circuits. Thus, the benefit of the CLGN model in saving regeneration circuits, compared with the GNTR model, is substantial.

\section{RSA with Limited Regeneration Nodes}

In this scenario, we simulate the RSA problem with a limited number of nodes that can be assigned as regeneration nodes. As we illustrate above, regeneration nodes are a limited resource because the allocation of regeneration nodes, as well as their maintenance, is very expensive. Consequently, regeneration nodes should be carefully allocated in the RSA problem. From the perspective of industry, the number of regeneration nodes is limited by the network implementation budget.

Hence, in this simulation scenario, we fix the maximum number of regeneration nodes. We maintain the optimization objective, $C+\varepsilon T$, for both the GNTR model and the CLGN model, and solve the RSA problem using the SA algorithm. We simulate this scenario in order to compare the performance of the total spectrum usage between the GNTR model and the CLGN model with limited regeneration nodes. In addition, compared with the results on the total spectrum usage from Section 5.3.1 (minimizing $C+\varepsilon T$ without constraining the number of regeneration nodes), we can observe the effects brought by utilizing more regeneration nodes. After simulating different cases, such $T \leq 2, T \leq 3$, and $T \leq 4$, the case of $T \leq 2$ is most representative. In this scenario, because of the QoT requirements, we stop simulation when the number of demands exceeds 30 . We maintain the simulation settings used in the previous scenarios (Section 5.3.1).

In Figure 5.7 (a), for the $T \leq 2$ case, the total spectrum usage required by the CLGN model is less than that of the GNTR model. In addition, the spectrum usage with the limited number of regeneration nodes is higher than the spectrum usage without the limitation on regeneration nodes. We conclude that the CLGN model 


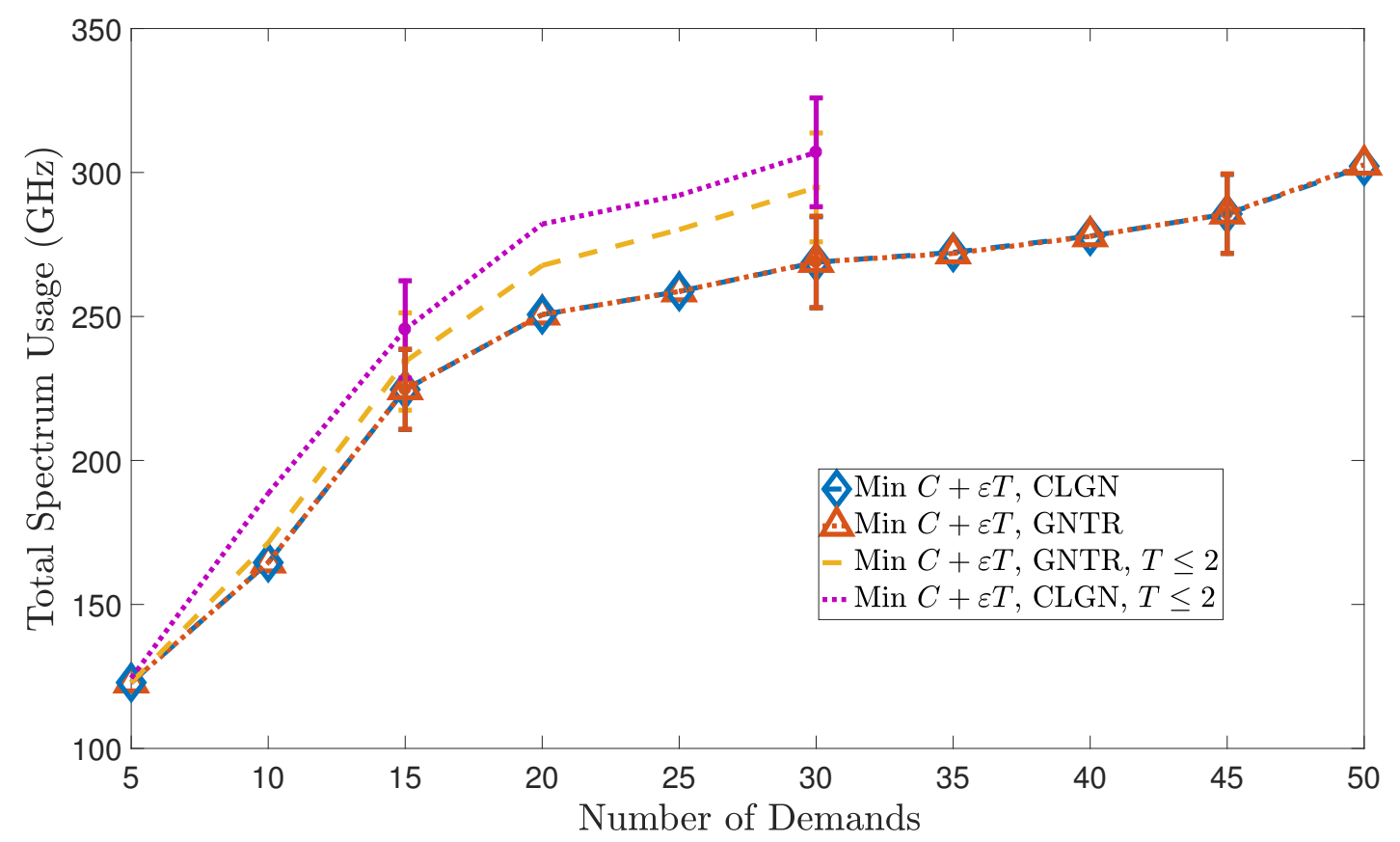

(a)

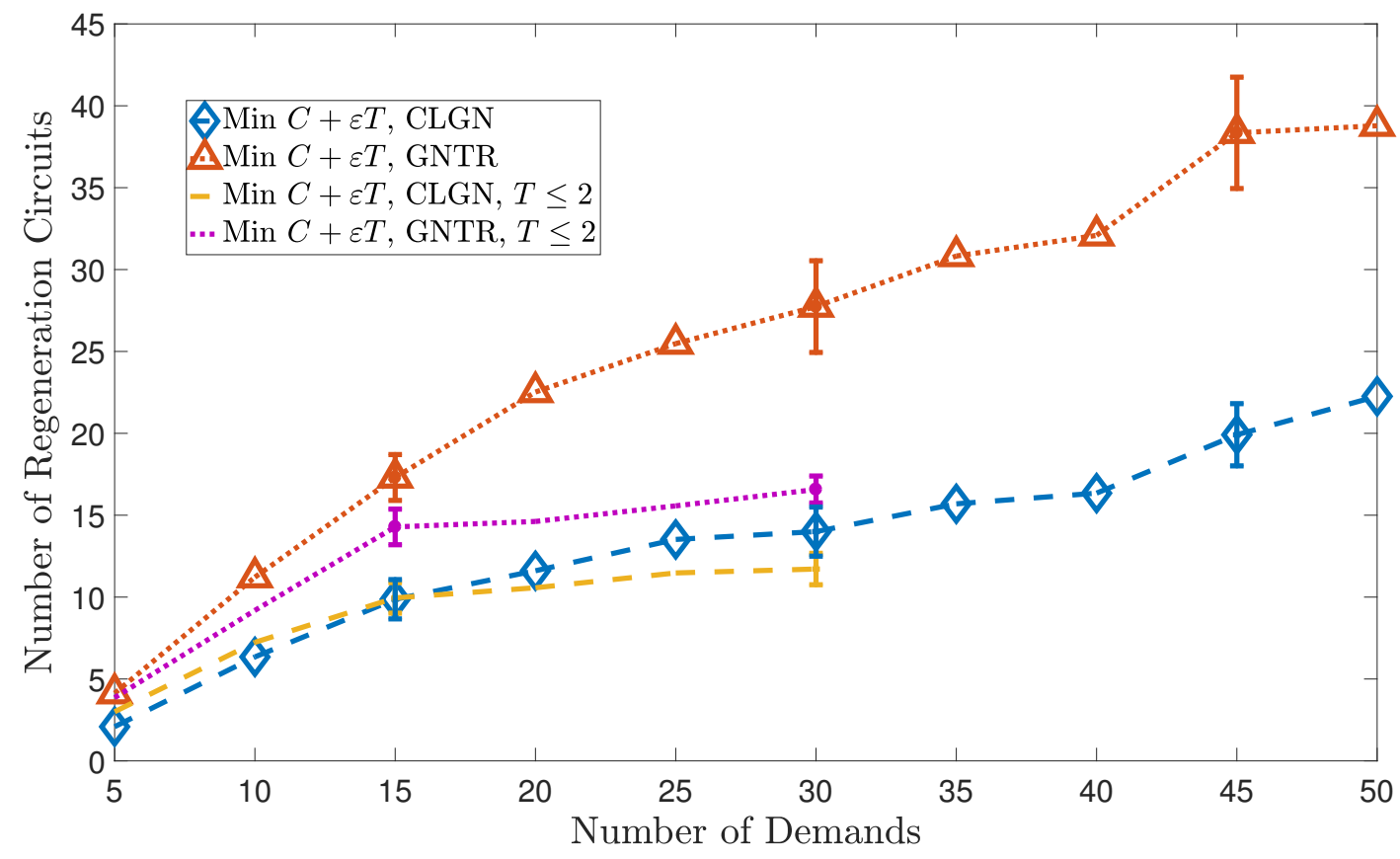

(b)

Figure 5.7: (a) Total spectrum usage versus number of demands with limited regeneration nodes, i.e., $T \leq 2$ and without limitation of regeneration nodes in NSF-24, with QPSK modulation. (b) Number of regeneration circuits versus number of demands with limited regeneration nodes, i.e., $T \leq 2$ and without limitation of regeneration nodes in NSF-24, with QPSK modulation. 
requires less spectrum, compared with the GNTR model, when there is a limited number of regeneration nodes. If we consider the result without the constraint on the number of regeneration nodes as a reference, we see that utilizing more regeneration nodes can save total spectrum usage. In Figure 5.7 (b), for $T \leq 2$, the number of regeneration circuits required by the CLGN model is less than that of the GNTR model. We thus conclude that the CLGN model is better in saving network resources compared with the GNTR model, when there is a limited number of regeneration nodes.

\subsubsection{Comparison with the Recursive MILP}

In this section, we compare the performance of our proposed heuristic algorithm, the SA, with another published algorithm, the re-MILP [2]. The optimization objective is to minimize $C+\varepsilon T$. We simulate and compare the performance of both the CLGN and the GNTR models solved by the SA algorithm and the re-MILP algorithm separately in the NSF-24 topology, with QPSK modulation. We again maintain the simulation settings used in the previous scenarios (Section 5.3.1).

The spectrum usage of both methods is shown in Figure 5.8. Compared with the re-MILP algorithm, the SA algorithm achieves a significant spectrum efficiency gain, which increases as the number of demands grows and reaches $19.0 \%$ at 50 demands. The spectrum usage of the CLGN model and the GNTR model is similar since the primary optimization objective here is the spectrum usage. The optimization thus sacrifices other resources such as regeneration nodes to ensure the optimality of the spectrum.

The usage of regeneration nodes and circuits is illustrated in Figures 5.9 and 5.10, respectively. For both resources, the optimization solution from the SA algorithm is better than that of the re-MILP algorithm in all cases. The number of regeneration 


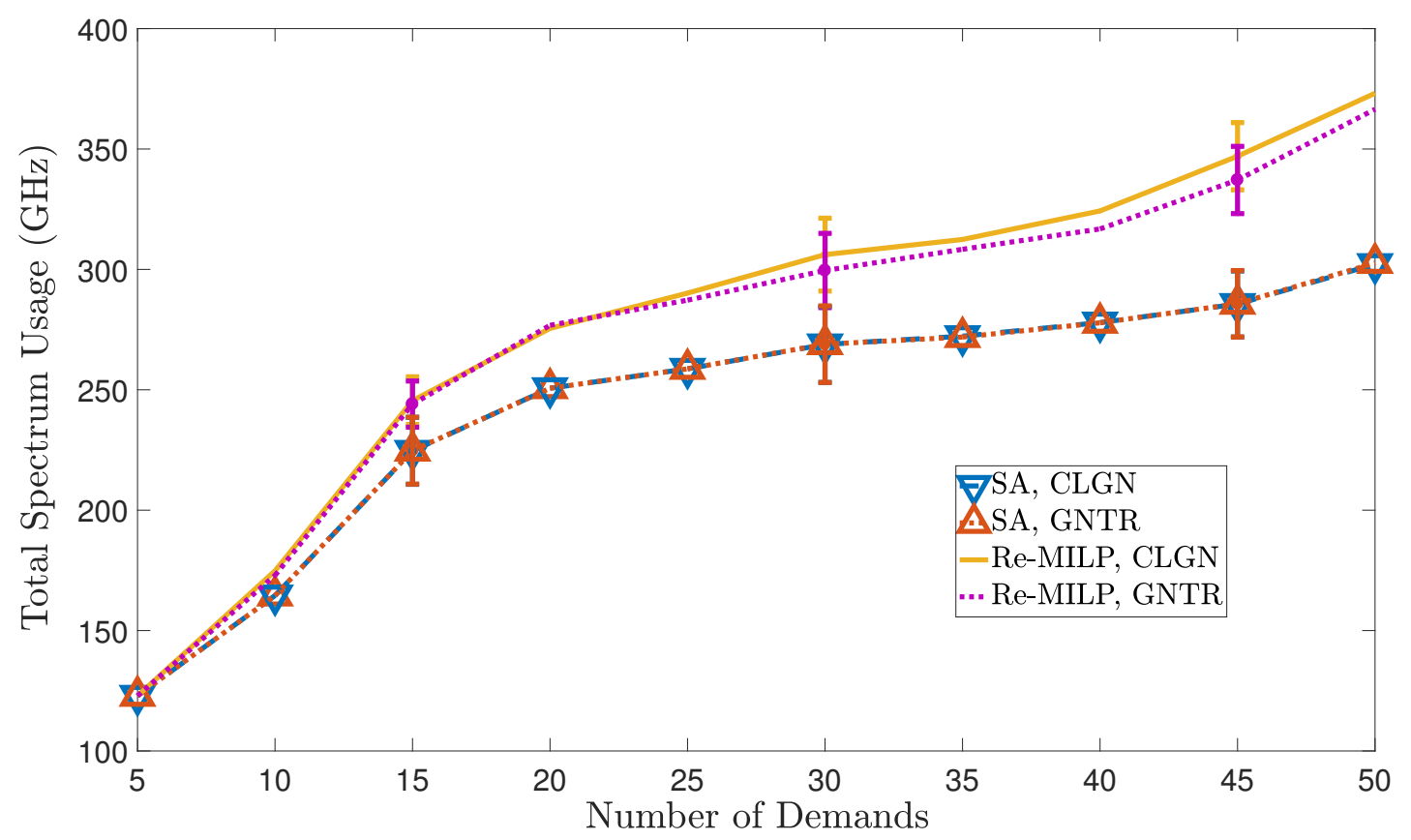

Figure 5.8: Total spectrum usage versus the number of demands for the SA and the re-MILP in NSF-24, with QPSK modulation.

nodes required by the SA algorithm is up to $28.5 \%$ lower than that of re-MILP when there are 50 demands. In addition, the number of regeneration circuits needed by the SA algorithm is up to $38.8 \%$ lower than that of the re-MILP algorithm at 50 demands. The reason is that the SA algorithm can avoid local optima in the optimization process and iteratively pursues a better result. The re-MILP algorithm uses the local optimum from the previous iteration as the starting point for the next iteration and, thus, yields a larger optimality gap. Moreover, the advantage of the SA algorithm over the re-MILP algorithm increases when the complexity of the RSA problem increases. Figures 5.9 and 5.10 also show that using the CLGN, a statedependent PLI model, instead of a worst-case constraint such as the GNTR, can significantly reduce the number of regeneration nodes and circuits required. Notably, the numbers of regeneration nodes and regeneration circuits of the SA algorithm with the CLGN model are $37.1 \%$ and $56.8 \%$, respectively, less than those of the re-MILP 




Figure 5.9: The number of regeneration nodes versus the number of demands for the SA and the re-MILP in NSF-24, with QPSK modulation.

with the GNTR model. In general, great savings of network resources are brought by the utilization of the SA algorithm with the CLGN model. 


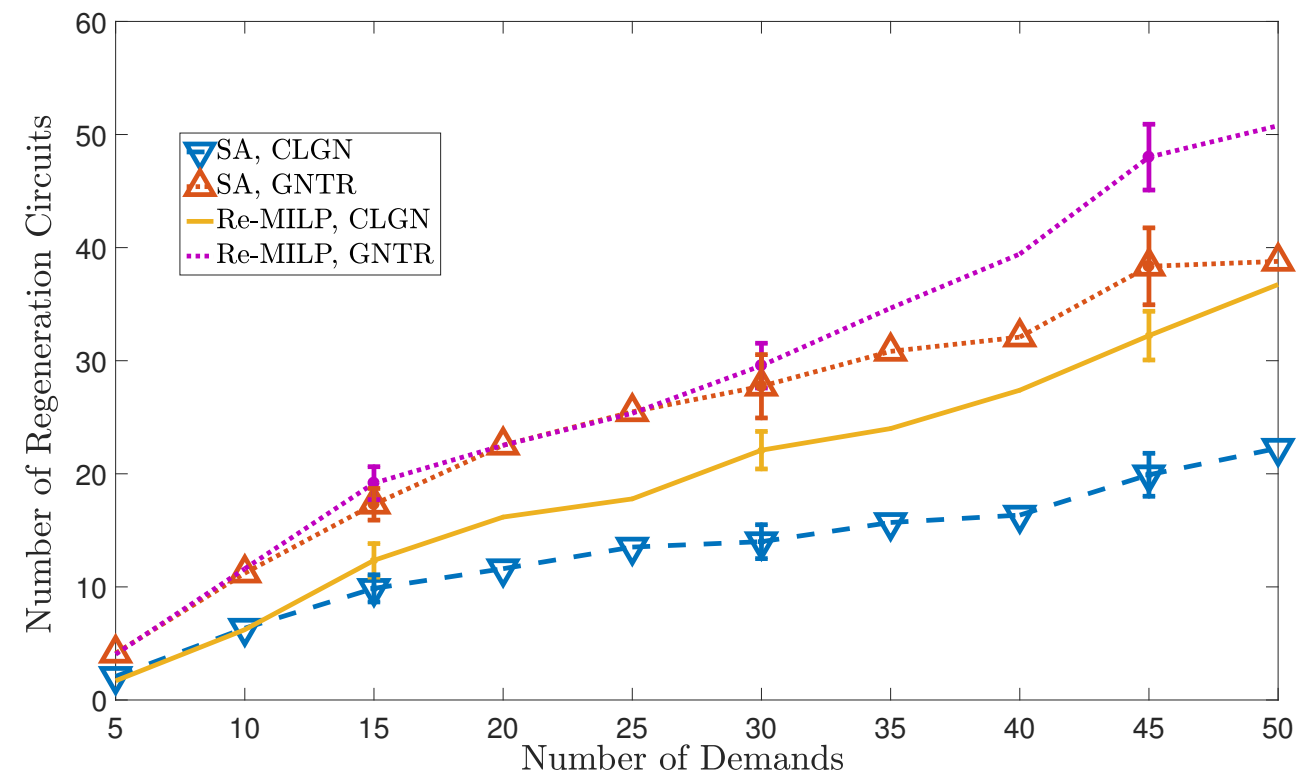

Figure 5.10: The number of regeneration circuits versus the number of demands for the SA and the re-MILP in NSF-24, with QPSK modulation. 


\section{Chapter 6}

\section{Conclusions and Future Work}

\subsection{Conclusions}

In this thesis we describe the RSA problem for EONs with PLIs and introduce the standard GN model and the TR model. Based on the standard GN model, we propose a novel estimation of PLIs, the CLGN model. In addition, we implement a GN-based analytic algorithm, referred to as the GNTR model, in order to make the comparison with the CLGN model fair.

The RSA problem suffering from PLIs is then modeled in MILP formulations. We propose a heuristic algorithm, referred to as the SA algorithm, to solve the RSA problem. We show through simulation that the CLGN model is better than the GNTR model in estimating PLIs. The better estimation of PLIs brought by the CLGN model saves resources of EONs, namely, the spectrum, regeneration nodes and regeneration circuits. Moreover, the proposed heuristic algorithm, the SA algorithm, outperforms the published re-MILP algorithm [2]. Utilizing our SA algorithm saves up to $38.8 \%$ of network resources compared with the re-MILP. Additionally, the utilization of the SA algorithm with the CLGN model increases these savings up to $56.8 \%$. We also 
compare our proposed system based on the CLGN model solved by the SA algorithm with the published system in [3]. Our algorithm can be used on larger scale networks and assign $80 \%$ more demands than the algorithm in [3]. We conclude that the proposed system is better at saving computational resources.

\subsection{Future Work}

In order to further improve the performance of our proposed work, we can consider the combination of utilizing the CLGN and the GNTR models. This combination would enable a further network resources saving. In addition, we plan to study a more practical scenario that there are more and more demands (such as $23 \times 24$ demands) deployed in the EON. 


\section{Bibliography}

[1] O. Gerstel, M. Jinno, A. Lord, and S. Yoo, "Elastic optical networking: A new dawn for the optical layer?" IEEE Communications Magazine, vol. 50, no. 2, pp. 12-20, 2012.

[2] X. Wang, M. Brandt-Pearce, and S. Subramaniam, "Impact of wavelength and modulation conversion on transluscent elastic optical networks using MILP," Journal of Optical Communications and Networking, vol. 7, no. 7, pp. 644-655, 2015.

[3] L. Yan, E. Agrell, M. N. Dharmaweera, and H. Wymeersch, "Joint assignment of power, routing, and spectrum in static flexible-grid networks," Journal of Lightwave Technology, vol. 35, no. 10, pp. 1766-1774, May 2017.

[4] J. Zhao, H. Wymeersch, and E. Agrell, "Nonlinear impairment-aware static resource allocation in elastic optical networks," IEEE Journal of Lightwave Technology, vol. 33, no. 22, pp. 4554-4564, 2015.

[5] L. Yan, "Resource allocation in flexible-grid optical networks with nonlinear interference," Ph.D. dissertation, Chalmers University of Technology, December 2015.

[6] L. Yan, E. Agrell, H. Wymeersch, P. Johannisson, R. Di Taranto, and M. BrandtPearce, "Link-level resource allocation for flexible-grid nonlinear fiber-optic com- 
munication systems," IEEE Photonics Technology Letters, vol. 27, no. 12, pp. 1250-1253, June 2015.

[7] Spectral grids for WDM applications: DWDM frequency grid, Telecommunication Standardization Sector of International Telecommunication Union Recommendation ITU-T G.694.1, 2012.

[8] L. C. Resendo, "Optimal approach for electronic grooming, routing and spectrum allocation in elastic optical networks," in Microwave and Optoelectronics Conference (IMOC), 2015 SBMO/IEEE MTT-S International. IEEE, 2015, pp. $1-5$.

[9] B. C. Chatterjee, N. Sarma, and E. Oki, "Routing and spectrum allocation in elastic optical networks: a tutorial," IEEE Communications Surveys $\&$ Tutorials, vol. 17, no. 3, pp. 1776-1800, 2015.

[10] J. Zhao, H. Wymeersch, and E. Agrell, "Nonlinear impairment aware resource allocation in elastic optical networks," in Optical Fiber Communication Conference. Optical Society of America, 2015, pp. M2I-1.

[11] P. Johannisson and E. Agrell, "Modeling of nonlinear signal distortion in fiberoptic networks," IEEE Journal of Lightwave Technology, vol. 32, no. 23, pp. 3942-3950, 2014.

[12] L. Yan, E. Agrell, H. Wymeersch, and M. Brandt-Pearce, "Resource allocation for flexible-grid optical networks with nonlinear channel model," Journal of Optical Communications and Networking, vol. 7, no. 11, pp. B101-B108, 2015.

[13] A. Klekamp, R. Dischler, and F. Buchali, "Limits of spectral efficiency and transmission reach of optical-OFDM superchannels for adaptive networks," IEEE Photonics Technology Letters, vol. 23, no. 20, pp. 1526-1528, 2011. 
[14] P. Johannisson and M. Karlsson, "Perturbation analysis of nonlinear propagation in a strongly dispersive optical communication system," IEEE Journal of Lightwave Technology, vol. 31, no. 8, pp. 1273-1282, 2013.

[15] P. Poggiolini, G. Bosco, A. Carena, V. Curri, Y. Jiang, and F. Forghieri, "The GN-model of fiber non-linear propagation and its applications," IEEE Journal of Lightwave Technology, vol. 32, no. 4, pp. 694-721, 2014.

[16] K. Christodoulopoulos, I. Tomkos, and E. Varvarigos, "Elastic bandwidth allocation in flexible OFDM-based optical networks," IEEE Journal of Lightwave Technology, vol. 29, no. 9, pp. 1354-1366, 2011.

[17] C. Dzongang, P. Galinier, and S. Pierre, "A tabu search heuristic for the routing and wavelength assignment problem in optical networks," IEEE Communications letters, vol. 9, no. 5, pp. 426-428, 2005.

[18] R. Ramaswami and K. N. Sivarajan, "Routing and wavelength assignment in all-optical networks," IEEE/ACM Transactions on Networking (TON), vol. 3, no. 5, pp. 489-500, 1995.

[19] H. Zang, J. P. Jue, B. Mukherjee et al., "A review of routing and wavelength assignment approaches for wavelength-routed optical WDM networks," Optical Networks Magazine, vol. 1, no. 1, pp. 47-60, 2000.

[20] D. J. Blumenthal, B.-E. Olsson, G. Rossi, T. E. Dimmick, L. Rau, O. Lavrova, R. Doshi, O. Jerphagnon, J. E. Bowers, V. Kaman et al., "All-optical label swapping networks and technologies," Journal of Lightwave Technology, vol. 18, no. 12 , p. $2058,2000$.

[21] S. Ramamurthy and B. Mukherjee, "Survivable WDM mesh networks. part iprotection," in INFOCOM'99. Eighteenth Annual Joint Conference of the IEEE 
Computer and Communications Societies. Proceedings. IEEE, vol. 2. IEEE, 1999, pp. $744-751$.

[22] B. Mukherjee, "WDM optical communication networks: progress and challenges," IEEE Journal on Selected Areas in Communications, vol. 18, no. 10, pp. 1810-1824, 2000.

[23] X. Yang and B. Ramamurthy, "Sparse regeneration in translucent wavelengthrouted optical networks: Architecture, network design and wavelength routing," Photonic Network Communications, vol. 10, no. 1, pp. 39-53, 2005.

[24] G. Shen and R. S. Tucker, "Translucent optical networks: the way forward [topics in optical communications]," IEEE Communications Magazine, vol. 45, no. 2, pp. 48-54, 2007.

[25] M. A. Ezzahdi, S. Al Zahr, M. Koubàa, N. Puech, and M. Gagnaire, "LERP: a quality of transmission dependent heuristic for routing and wavelength assignment in hybrid WDM networks," in Computer Communications and Networks, ICCCN 2006. Proceedings. 15th International Conference on. IEEE, 2006, pp. $125-136$.

[26] M. Flammini, A. Marchetti-Spaccamela, G. Monaco, L. Moscardelli, and S. Zaks, "On the complexity of the regenerator placement problem in optical networks," IEEE/ACM Transactions on Networking (TON), vol. 19, no. 2, pp. 498-511, 2011.

[27] K. Christodoulopoulos, I. Tomkos, and E. A. Varvarigos, "Routing and spectrum allocation in OFDM-based optical networks with elastic bandwidth allocation," in Global Telecommunications Conference (GLOBECOM 2010). IEEE, 2010, pp. 1-6. 
[28] M. Klinkowski and K. Walkowiak, "A heuristic algorithm for routing, spectrum, transceiver and regeneration allocation problem in elastic optical networks," in Transparent Optical Networks (ICTON), 2016 18th International Conference on. IEEE, 2016, pp. 1-4.

[29] M. Jinno, H. Takara, B. Kozicki, Y. Tsukishima, Y. Sone, and S. Matsuoka, "Spectrum-efficient and scalable elastic optical path network: architecture, benefits, and enabling technologies," IEEE Communications Magazine, vol. 47, no. 11, 2009.

[30] M. Rouse. (2013) Backbone. [Online]. Available: http://searchtelecom. techtarget.com/definition/backbone

[31] X. Wang, "Resource assignment for fiber optic networks," Ph.D. dissertation, University of Virginia, November 2014.

[32] L. Yan, E. Agrell, and H. Wymeersch, "Resource allocation in nonlinear flexiblegrid fiber-optic networks," in Proc. Optical Fiber Communication Conference (OFC), Los Angeles, CA, Mar. 2015, pp. Tu2I-5.

[33] B. Mukherjee, Optical WDM networks. Springer Science \& Business Media, 2006.

[34] M. Klinkowski and K. Walkowiak, "Routing and spectrum assignment in spectrum sliced elastic optical path network," IEEE Communications Letters, vol. 15, no. 8, pp. 884-886, 2011.

[35] M. Ruiz, M. Żotkiewicz, L. Velasco, and J. Comellas, "A column generation approach for large-scale RSA-based network planning," in Transparent Optical Networks (ICTON), 2013 15th International Conference on. IEEE, 2013, pp. 1-4. 
[36] Wikipedia. (2017) Linear programming. [Online]. Available: https://en. wikipedia.org/wiki/Linear_programming

[37] L. R. Costa and A. C. Drummond, "New distance-adaptive modulation scheme for elastic optical networks," IEEE Communications Letters, vol. 21, no. 2, pp. 282-285, 2017.

[38] P. Datta, M. Sridharan, and A. K. Somani, "A simulated annealing approach for topology planning and evolution of mesh-restorable optical networks," in 8th IFIP Working Conference on Optical Networks Design and Modeling (ONDM), vol. 16. Citeseer, 2003.

[39] A. Rodriguez, A. Gutierrez, L. Rivera, and L. Ramirez, "RWA: Comparison of genetic algorithms and simulated annealing in dynamic traffic," in Advanced Computer and Communication Engineering Technology. Springer, 2015, pp. $3-14$.

[40] Q. Wang and L. K. Chen, "Performance analysis of multicast traffic over spectrum elastic optical networks," in Optical Fiber Communication Conference. Optical Society of America, 2012, pp. OTh3B-7.

[41] D. J. Ives, A. Alvarado, and S. J. Savory, "Adaptive transceivers in nonlinear flexible networks," in ECOC 2016; 42nd European Conference on Optical Communication, Sept 2016, pp. 1-3.

[42] D. J. Ives, P. Bayvel, and S. J. Savory, "Physical layer transmitter and routing optimization to maximize the traffic throughput of a nonlinear optical mesh network," in Proc. IEEE International Conference of Optical Network Design and Modeling (ONDM), Stockholm, Sweden, May 2014, pp. 168-173. 
[43] MathWorks. (2013) Histogram properties. [Online]. Available: http://www. leandro-coelho.com/linearization-product-variables/

[44] L. C. Coelho. (2017) Linearization of the product of two variables. [Online]. Available: https://www.mathworks.com/help/matlab/ref/histogram-properties.html

[45] Rivanna Research Computing Cluster , "Rivanna research computing cluster," http://arcs.virginia.edu/rivanna, 2017.

[46] Gurobi Optimization, Inc., "Gurobi optimizer reference manual," http://www. gurobi.com, 2015.

[47] Z. Fan, Y. Li, G. Shen, and C. K. C. Chan, "Distance-adaptive spectrum resource allocation using subtree scheme for all-optical multicasting in elastic optical networks," Journal of Lightwave Technology, vol. 35, no. 9, pp. 1460-1468, May 2017. 